\title{
Flexible Spline Based Models for the Analysis of Panel Data Under a Markov Assumption
}

\section{Jonathan Boudreau}

\author{
A Thesis submitted to \\ the Faculty of Graduate Studies and Research \\ in partial fulfilment of \\ the requirements for the degree of \\ Master of Science \\ in
}

\begin{abstract}
Mathematics
Probability and Statistics

Carleton University

Ottawa, Ontario, Canada
\end{abstract}

April 2014

Copyright (C)

2014 - Jonathan Boudreau 
The undersigned recommend to

the Faculty of Graduate Studies and Research

acceptance of the Thesis

\title{
Flexible Spline Based Models for the Analysis of Panel Data Under a Markov Assumption
}

\author{
Submitted by Jonathan Boudreau \\ in partial fulfilment of the requirements for the degree of \\ Master of Science
}

Dr. Jason Nielsen,

Supervisor

Dr. Patrick Farrell, Carleton University, School of Mathematics and Statistics

Dr. Paul Villeneuve, Carleton University, Department of Health Sciences

Dr. Mahmoud Zarepour, University of Ottawa, Department of Mathematics and Statistics

Carleton University

2014 


\section{Abstract}

Panel data is encountered in a large variety of disciplines, from social sciences to medical studies, and has been used to examine increasingly complex processes. Recent work in the analysis of panel data, particularly under Markov assumptions, has been leading towards models for data that evolve over time. We propose a method for modeling such non-homogeneous processes through the use of splines and penalized splines. We provide a brief overview of spline theory, as well as the basic notions for modeling panel data under a Markov assumption. We then discuss the proposed method and supply examples from simulated and previously modeled data. The proposed method is particularly well suited to exploratory analysis and simplifying complex models. 


\section{Table of Contents}

Abstract $\quad$ iii

Table of Contents $\quad$ iv

List of Tables $\quad$ vi

List of Figures $\quad$ vii

1 Introduction 1

2 Background Information 2

2.1 Panel Data . . . . . . . . . . . . . . . . . . 2

2.2 Continuous Time Markov Chains . . . . . . . . . . . . . . . 3

2.3 Splines ............................. 7

2.4 B-Splines . . . . . . . . . . . . . . . . . . . . . . . . . 8

2.5 Penalized Splines and Smoothing . . . . . . . . . . . . . . . . 12

2.5.1 Lower Order Splines . . . . . . . . . . . . . . . . . . 12

2.5.2 Modifing the Number and Location of Knots . . . . . . . . . . 13

2.5.3 Regularization . . . . . . . . . . . . . . . 13

2.5.4 P-Splines .......................... 15

2.6 Selecting the Smoothing Parameter . . . . . . . . . . . . 17 
$\begin{array}{llr}3 & \text { Model } & 20\end{array}$

3.1 Spline Notation . . . . . . . . . . . . . . . . . 20

3.2 Transition Probabilities . . . . . . . . . . . . . . . . . 22

3.3 Transition intensities . . . . . . . . . . . . . . . 23

3.4 Expressing Transition Probabilities and Intensities . . . . . . . . . . . 24

3.5 Maximum Likelihood Estimation . . . . . . . . . . . . 26

3.6 Penalized Maximum Likelihood Estimation . . . . . . . . . . . . . . . 28

3.7 Derived Quantities . . . . . . . . . . . . . . 29

3.8 Extensions . . . . . . . . . . . . . . . . . . . . . . . 30

3.8.1 Covariates ........................ 30

3.9 Embedability and Estimability . . . . . . . . . . . . . . 31

4 Results

4.1 Simulation . . . . . . . . . . . . . . . . . . 33

4.1 .1 B-Splines .......................... 34

$4.1 .2 \quad$ P-Splines . . . . . . . . . . . . . . . . 47

4.2 Examples ............................ 61

4.2.1 Kabfleisch \& Lawless . . . . . . . . . . . . . . . . . . . . . 61

4.2.2 Titman .................... 64

$\begin{array}{llr}5 & \text { Conclusion } & 67\end{array}$

$\begin{array}{ll}\text { List of References } & 68\end{array}$ 


\section{List of Tables}

1 Parameter Values for the Kalbfleisch and Lawless Data . . . . . . . . 62 


\section{List of Figures}

1 B-Spline Basis for Unique Knots . . . . . . . . . . . . . . . . . . 10

2 B-Spline Basis for Non-unique Knots . . . . . . . . . . . . . . 11

3 Example B-Spline With Basis Functions _. . . . . . . . . . . . 12

4 B-Spline Estimates for Simulated Data With 10 Knots and $N=200$. 35

5 B-Spline Estimates for Simulated Data With 25 Knots and $N=200$. 36

6 B-Spline Estimates for Simulated Data With 50 Knots and $N=200$. 37

$7 \quad$ B-Spline Estimates for Simulated Data With 10 Knots and $N=500$. 38

8 B-Spline Estimates for Simulated Data With 25 Knots and $N=500$. 39

9 B-Spline Estimates for Simulated Data With 50 Knots and $N=500$. 40

10 B-Spline Variances for Simulated Data With 10 Knots and $N=200$. 41

11 B-Spline Variance for Simulated Data With 25 Knots and $N=200$. 42

12 B-Spline Variances for Simulated Data With 50 Knots and $N=200$. 43

13 B-Spline Variances for Simulated Data With 10 Knots and $N=500$. 44

14 B-Spline Variances for Simulated Data With 25 Knots and $N=500$. 45

15 B-Spline Variances for Simulated Data With 50 Knots and $N=500$. 46

16 P-Spline Estimates for Simulated Data With 10 Knots and $N=200$. 49

17 P-Spline Estimates for Simulated Data With 25 Knots and $N=200$. 50

18 P-Spline Estimates for Simulated Data With 50 Knots and $N=200$. 51

19 P-Spline Estimates for Simulated Data With 10 Knots and $N=500$. 52

20 P-Spline Estimates for Simulated Data With 25 Knots and $N=500$. 53 
21 P-Spline Estimates for Simulated Data With 50 Knots and $N=500$. 54

22 P-Spline Variances for Simulated Data With 10 Knots and $N=200 \quad . \quad 55$

23 P-Spline Variances for Simulated Data With 25 Knots and $N=200$. 56

24 P-Spline Variances for Simulated Data With 50 Knots and $N=200$. 57

25 P-Spline Variances for Simulated Data With 10 Knots and $N=500$. 58

26 P-Spline Variances for Simulated Data With 25 Knots and $N=500$. 59

27 P-Spline Variances for Simulated Data With 50 Knots and $N=500$. 60

28 Transition Intensities for the Kalbfleisch and Lawless Data . . . . . . 63

29 Transition Intensities for the Titman Data . . . . . . . . . . . . 66 


\section{Chapter 1}

\section{Introduction}

Panel data is encountered in a large variety of disciplines, from social sciences to medical studies, and everything in between. The analysis of panel data examines time dependent processes, often under a Markov assumption, through the classification of observed individuals in various states. Of particular interest are the rates at which individuals transition from one state to another, and whether or not these tendencies remain constant over time. Focusing primarily on the non-constant, or non timehomogeneous, case, we propose a method using splines and penalized splines for modeling panel data.

We begin our discussion by reviewing background material in Section 2, covering topics such as panel data, continuous-time Markov chains, splines, basis functions, and regularization. We then outline the main elements of the proposed method in Section 3, which discusses transition probabilities and intensities, maximum likelihood estimation, derived values, as well as extensions and limitations. Section 4 concludes our discussion by presenting examples for simulated and previously modeled data. 


\section{Chapter 2}

\section{Background Information}

The following is an introduction that will serve as a basis for our discussion. The covered topics include panel data, continuous-time Markov chains, splines, basis functions, and regularization.

\subsection{Panel Data}

Panel data refers to the longitudinal information gathered by observing a particular set of individuals over a given period of time. This type of data is concerned with time dependent events, specifically those which are recurrent in nature. Panel data is presented as a series of observations at discrete time points, between which it is assumed that no information is known (Kalbfleisch and Lawless, 1985). Despite the fact that time points of observation are discrete, the process of interest is assumed to be continuous. Data sets with these characteristics may be modeled using continuous time Markov chains, under a Markov assumption. 


\subsection{Continuous Time Markov Chains}

Under a Markov assumption, a continuous-time stochastic process is divided into $k$ exhaustive and mutually exclusive states that make up the state space $\mathcal{S}=\{1,2, \ldots, k\}$. The process is said to be a Markov chain if the transitions between states follow the Markov property, by which the probability of occupying a future state, given the previous states, depends only on the current state (Bhat, 1984).

If an individual can transition from a given state to another, the former is said to be accessible from the latter. If two states are mutually accessible, they are said to communicate. States that communicate, form an equivalence relation, and may be grouped into an equivalence class. If all the states of a Markov process belonging to one equivalence class, they are said to be irreducible (Bhat, 1984).

States for which the probabilities of occupation become effectively 0 as $t \rightarrow \infty$ are transient, whereas states with probabilities equal to 1 are absorbing. If a state has a strictly positive probability in the limit, it is called positive recurrent (Bhat, 1984).

If a state is recurrent, the minimum elapsed time necessary for an individual to return to that state is called the period. If individuals can return to the same state within one period, the process is aperiodic. Markov processes that are positive recurrent and aperiodic are termed ergodic (Bhat, 1984).

Markov chains that are aperiodic and irreducible admit a stationary limiting distribution (Bhat, 1984). Stationary distributions, as their name implies, are constant over time. This means that while transitions between states continue to occur, the probability of occupying a given state remains the same, regardless of the elapsed time. 
Letting $X(t)$ be the state occupied by an individual at time $t$, we define the transition probabilities from state $i$ at time $s$ to state $j$ at time $t$, for $0 \leq s \leq t$, as:

$$
p_{i j}(s, t)=P\{X(t)=j \mid X(s)=i\}
$$

for $i, j=1, \ldots, k$. If $k$ is finite, then $\boldsymbol{P}(s, t)=\left\{p_{i j}(s, t)\right\}_{k \times k}$ is a $k \times k$ transition probability matrix, whose elements are positive with the condition $\sum_{j} p_{i j}(s, t)=1$.

Using the properties of conditional probabilities as well as the Markov property, for $X(t), X(s), X(u) \in \mathcal{S}$ and $0 \leq s \leq u \leq t$, we obtain the following relations for all $i, j=1, \ldots, k$ (Bhat, 1984):

$$
\begin{aligned}
p_{i j}(s, t) & =P\{X(t)=j \mid X(s)=i\} \\
& =\sum_{k \in \mathcal{S}} P\{X(t)=j, X(u)=k \mid X(s)=i\} \\
& =\sum_{k \in \mathcal{S}} \frac{P\{X(t)=j, X(u)=k, X(s)=i\}}{P\{X(s)=i\}} \\
& =\sum_{k \in \mathcal{S}} \frac{P\{X(u)=k, X(s)=i\}}{P\{X(s)=i\}} \cdot \frac{P\{X(t)=j, X(u)=k, X(s)=i\}}{P\{X(u)=k, X(s)=i\}} \\
& =\sum_{k \in \mathcal{S}} P\{X(u)=k \mid X(s)=i\} P\{X(t)=j \mid X(u)=k, X(s)=i\} \\
& =\sum_{k \in \mathcal{S}} P\{X(u)=k \mid X(s)=i\} P\{X(t)=j \mid X(u)=k\} \\
& =\sum_{k \in \mathcal{S}} p_{i k}(s, u) p_{k j}(u, t) .
\end{aligned}
$$

The above are refered to as the Chapman-Kolmogorov equations and may be conveniently expressed in matrix notation as:

$$
\boldsymbol{P}(s, t)=\boldsymbol{P}(s, u) \boldsymbol{P}(u, t)
$$


These equations indicate that the transition probabilities between times $s$ and $t$ may be subdivided at any intermediary value $u$.

If the transition probabilities vary based on the values of $s$ and $t$, in addition to the elapsed time, $t-s$, the process is considered non time-homogeneous. By contrast, if the transition probabilities depend only on the elapsed time, $t-s$, the process is considered to be time-homogeneous. Note that for the time-homogeneous case $\boldsymbol{P}(s, t)=\boldsymbol{P}(0, t-s)$.

If we differentiate (2.2) with respect to $s$ for $u=s$, we obtain the Kolmogorov Backward equation:

$$
\boldsymbol{P}^{\prime}(s, t)=\tilde{\boldsymbol{Q}}(s) \boldsymbol{P}(s, t) .
$$

Similarly, if we differentiate (2.2) with respect to $t$ for $u=t$, we obtain the Kolmogorov Forward equation:

$$
\boldsymbol{P}^{\prime}(s, t)=\boldsymbol{P}(s, t) \tilde{\boldsymbol{Q}}(t)
$$

In both cases, $\tilde{\boldsymbol{Q}}(t)=\left\{\tilde{q}_{i j}(t)\right\}_{k \times k}$ is the intensity matrix, or infinitensimal generator, whose elements are:

$$
\tilde{q}_{i j}(t)= \begin{cases}\lim _{\Delta t \rightarrow 0} p_{i j}(t, t+\Delta t) / \Delta t & \text { for } i \neq j \\ -\sum_{j \neq i} \tilde{q}_{i j}(t) & \text { for } i=j .\end{cases}
$$

It will be of interest for us to integrate this value over a given interval $[s, t]$. Thus, we define the elements of $\boldsymbol{Q}(s, t)=\left\{q_{i j}(s, t)\right\}_{k \times k}$ as: 


$$
q_{i j}(s, t)=\int_{s}^{t} \tilde{q}_{i j}(u) d u
$$

To obtain a valid point estimate of the infinitessimal generator over $[s, t]$, we take the mean integrated value of the $\tilde{q}_{i j}(t)$ 's with respect to $[s, t]$. This produces the $\overline{\boldsymbol{Q}}(s, t)=\left\{\bar{q}_{i j}(s, t)\right\}_{k \times k}$ matrix:

$$
\bar{q}_{i j}(s, t)=\frac{q_{i j}(s, t)}{t-s}=\frac{\int_{s}^{t} \tilde{q}_{i j}(u) d u}{t-s} .
$$

Note that the time-homogeneous $\bar{q}_{i j}(s, t)$ 's are a special case of the nonhomogeneous intensities. Since they depend only on the elapsed time, $t-s$, and not on the particular values of $s$ or $t$, they may be simplified: $\bar{q}_{i j}(s, t)=\tilde{q}_{i j}(t-s)$.

Recall that (2.3) and (2.4) are systems of ordinary linear differential equations. They admit the unique solution (Kalbfleisch and Lawless, 1985):

$$
\boldsymbol{P}(s, t)=e^{\tilde{\boldsymbol{Q}}(s) \cdot(t-s)}=e^{\tilde{\boldsymbol{Q}}(t) \cdot(t-s)}=\sum_{u=0}^{\infty} \frac{\tilde{\boldsymbol{Q}}(t)^{u} \cdot(t-s)^{u}}{u !} .
$$

Replacing $\tilde{\boldsymbol{Q}}(t)$ with it's estimate over $[s, t], \overline{\boldsymbol{Q}}(s, t)$, we obtain:

$$
\boldsymbol{P}(s, t)=e^{\overline{\boldsymbol{Q}}(s, t) \cdot(t-s)}=e^{\boldsymbol{Q}(s, t)},
$$

which expresses $\boldsymbol{P}(s, t)$ as a function of the integrated intensity matrix, $\boldsymbol{Q}(s, t)$.

From (2.9) it is obvious that $\boldsymbol{Q}(s, t)$ is a reparameterization of $\boldsymbol{P}(s, t)$, since it is possible to use one or the other, and a given initial condition, to specify the entire stochastic process. 
Using a canonical decomposition, we may reformulate the relation between $\boldsymbol{P}(s, t)$ and $\boldsymbol{Q}(s, t)$. Letting $\boldsymbol{Q}(s, t)=\boldsymbol{A} \boldsymbol{D} \boldsymbol{A}^{-1}$, and $\boldsymbol{D}=\operatorname{diag}\left(d_{1}(s, t), d_{2}(s, t), \ldots, d_{k}(s, t)\right)$, where the $d_{i}(s, t)$ 's are eigenvalues of $\boldsymbol{Q}(s, t)$ and $\boldsymbol{A}$ is the matrix of coresponding eigenvectors, we may restate the relation as (Kalbfleisch and Lawless, 1985):

$$
\boldsymbol{P}(s, t)=\boldsymbol{A} \operatorname{diag}\left(e^{d_{1}(s, t)}, e^{d_{2}(s, t)}, \ldots, e^{d_{k}(s, t)}\right) \boldsymbol{A}^{-1}
$$

If the eigenvalues of $\boldsymbol{Q}(s, t)$ are all 0, then the Markov process has a stationary distribution. If the process is also irreducible, then the stationary distribution, $\vec{\pi}=\left\{\pi_{1}, \ldots, \pi_{k}\right\}_{k \times 1}$, for $\vec{\pi} \neq \overrightarrow{0}$, is an equilibrium distribution, such that $\vec{\pi}^{\top} \boldsymbol{Q}(s, t)=\overrightarrow{0}$ (Bhat, 1984). This is a very useful quantity, since the $\pi_{i}$ 's, if they exsist, are the unconditional probabilities of occupying the $i^{\text {th }}$ state.

\subsection{Splines}

The term "spline" originates from the flexible peices of wood used in the making of ship hulls (de Boor, 1978). Similarly, splines are flexible functions constructed from piecewise polynomials, called basis functions, with continuity conditions that force them to be equal to their neigbhours at joining points.

Joining points are characterized by the presence of one or more knots. Each knot relaxes a continuity condition imposed on the basis functions. At any given joining point, the number of continuity conditions imposed is equal to the order of the spline minus the number of knots. The order of a spline, denoted for convenience by $k+1$, is one plus the degree of the leading monomial of the basis functions. It is also the number of non-zero basis functions at any value between joining points. For instance, cubic splines use third degree polynomials in their basis and have an order of $3+1=4$. 
Splines are defined on open or closed intervals depending on the basis used and the assumptions made about their behaviour beyond the boundary points. The interval between the boundary points is subdivided by, say $n$, interior knots, whose unique values define the previously mentioned joining points. Additionally there is usually a loss of continuity near the boundaries. This is consistent with the idea that less is known about the underlying function outside of the the range of the data (de Boor, 1978).

In addition to basis functions and knots, splines are constructed using a series of coefficients. In effect, splines are linear combinations of basis functions and may be expressed as:

$$
f(x)=\sum_{i=1}^{n+k+1} \alpha_{i} \cdot B_{i}(x),
$$

where the $B_{i}(x)$ 's are the basis functions, the $\alpha_{i}$ 's are their coefficients, and $n$ is the number of interior knots (de Boor, 1978). These coefficients may be fit in the usual manner using least squares.

The wide use of splines is due mainly to their interesting numerical properties. For instance, splines: a) define a large class of flexible functions, b) are computationally efficient, c) are easily stored on a computer, and d) may be fit to data using least squares in the basic scatterplot smoothing context (de Boor, 1978).

\subsection{B-Splines}

The most popular spline basis spanning the spline space are the B-Spline basis introduced by de Boor (1978). The B-Spline basis functions of order $k+1$ use a series of $k+1$ boundary knots on either side of the $n$ interior knots, for a total of 
$n+2 k+2$ knots. Between these knots, $n+k+1$ basis functions are defined using divided differences ${ }^{1}$.

Fortunately, there exists an efficient recursive relation for generating the B-Spline basis. Letting the knots be denoted as $t_{i}$, for $i=-k,-(k-1), \ldots, 0,1,2, \ldots, n, n+$ $1, \ldots, n+k+1$, the recursive relation may be stated as (de Boor, 1978):

$$
B_{i, j}(x)=\frac{x-t_{i}}{t_{i+j-1}-t_{i}} B_{i, j-1}(x)+\frac{t_{i+j}-x}{t_{i+j}-t_{i+1}} B_{i+1, j-1}(x),
$$

where $B_{i, 0}(x)=1$ for all $x \in\left[t_{i}, t_{i+1}\right]$, and $B_{i, 0}(x)=0$ elsewhere. Thus, to construct a B-Spline basis of order $k+1$, we define the basis of order $1(j=0)$ and evaluate (2.12) $k$ times. This produces basis functions similar to those depicted in Figure 1.

De Boor remarks that since $x$ must be contained within the interval $\left[t_{i}, t_{i+k}\right]$, any term whose denominator is 0 will also be multiplied by 0 . Rather than consider this ratio as undefined, he suggests that we follow the rule of thumb that anything multiplied by 0 is 0 . This implies that multiple knots may be placed at a joining point, reducing its continuity at that point. For example, adding a second interior knot at the joining point $X_{3}$ in Figure 1 produces the basis functions shown in Figure 2.

The above allows us to define a basis with many interesting properties. In addition to those listed in section 2.3, B-Splines: a) are numerically stable, b) have compact support, c) have a banded structure, and d) may be computed recursively (de Boor, 1978). Figure 3 provides a visual example of a B-Spline and its basis functions multiplied by their respective coefficients.

\footnotetext{
${ }^{1}$ Equivalently, the B-Spline basis may expressed in the form of a truncated power series. Interested readers should consult de Boor (1978) or Eilers and Marx (2010) for more information, as this topic is beyond the scope of our current discussion.
} 


\section{Order 1 B-Spline Basis}

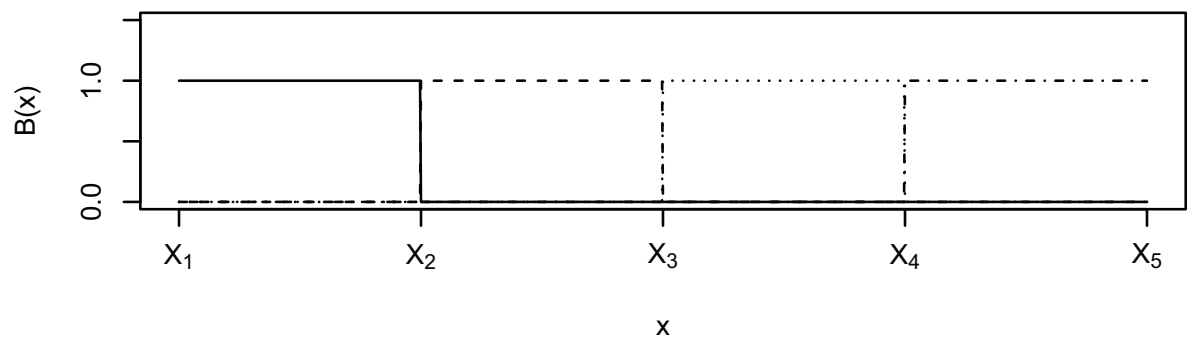

Order 2 B-Spline Basis

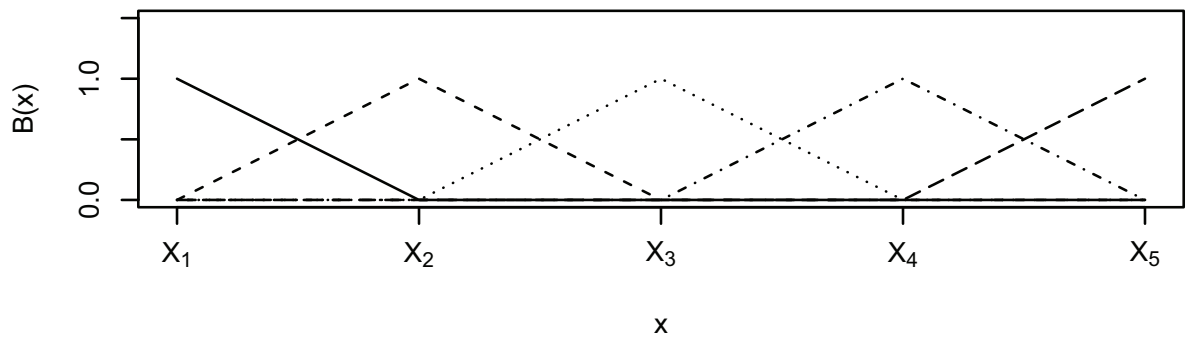

Order 3 B-Spline Basis

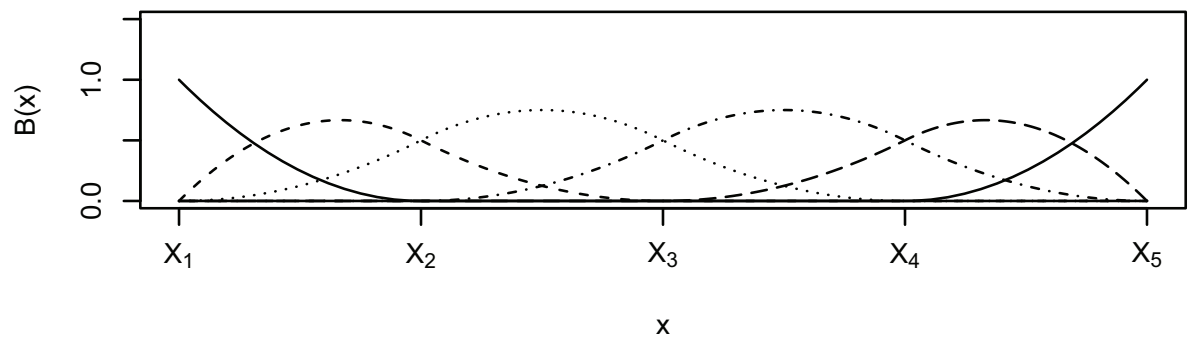

Order 4 B-Spline Basis

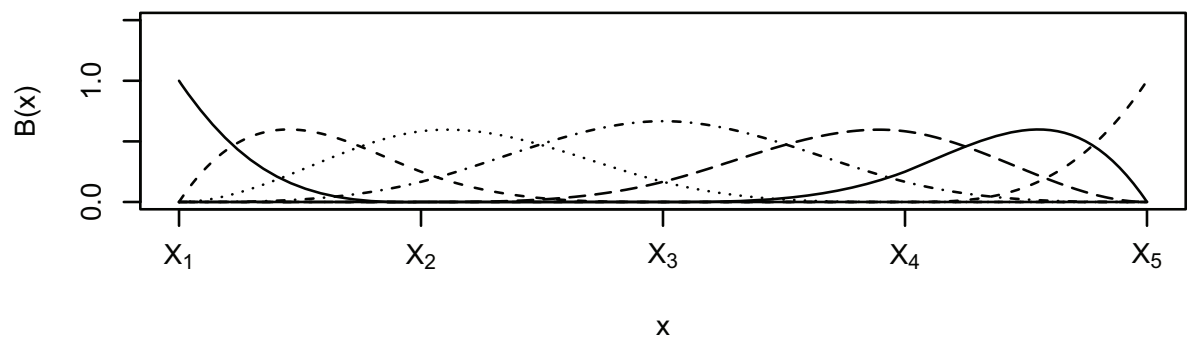

Figure 1: B-Spline basis functions for splines of orders 1-4, with interior knots positionned at $X_{2}, X_{3}$, and $X_{4}$. 


\section{Order 1 B-Spline Basis}

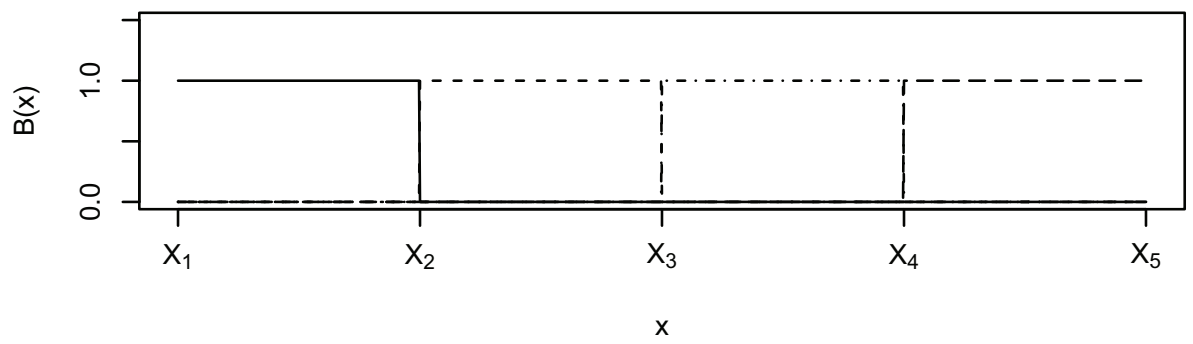

Order 2 B-Spline Basis

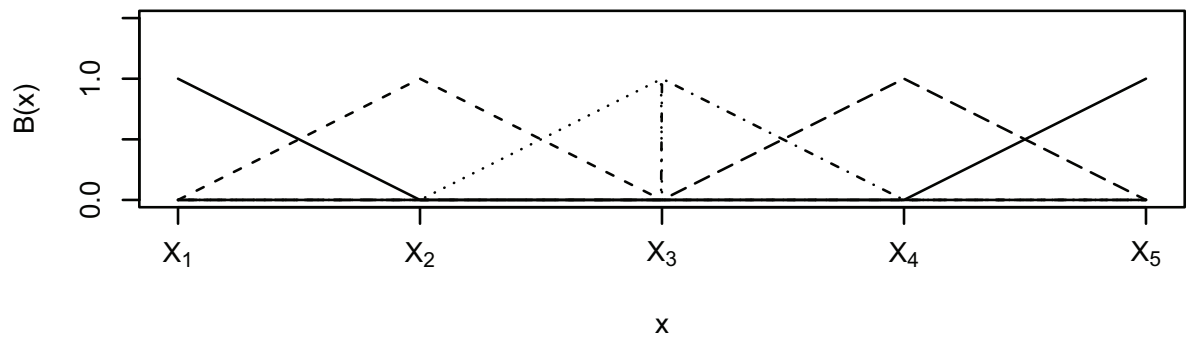

Order 3 B-Spline Basis

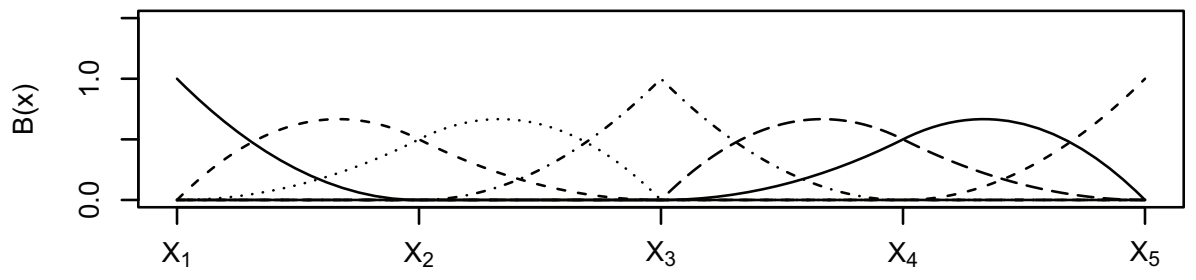

$\mathrm{x}$

Order 4 B-Spline Basis

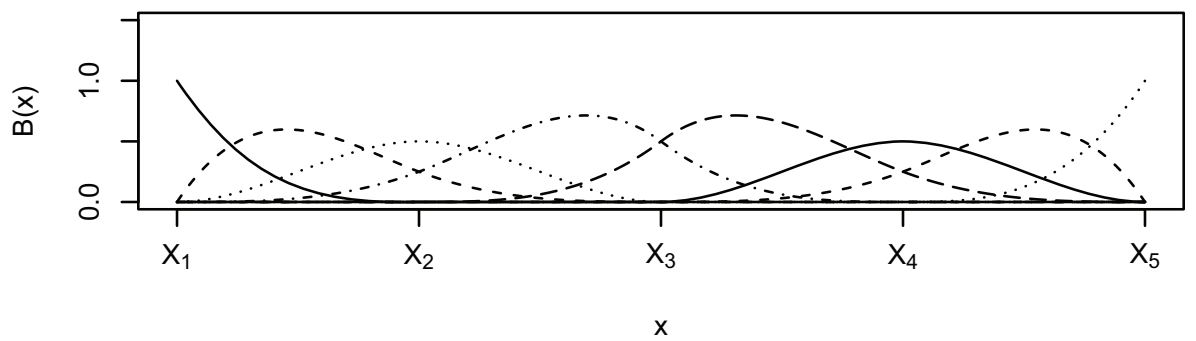

Figure 2: B-Spline basis functions for splines of orders 1-4, with interior knots positionned at $X_{2}, X_{3}, X_{3}$, and $X_{4}$. 


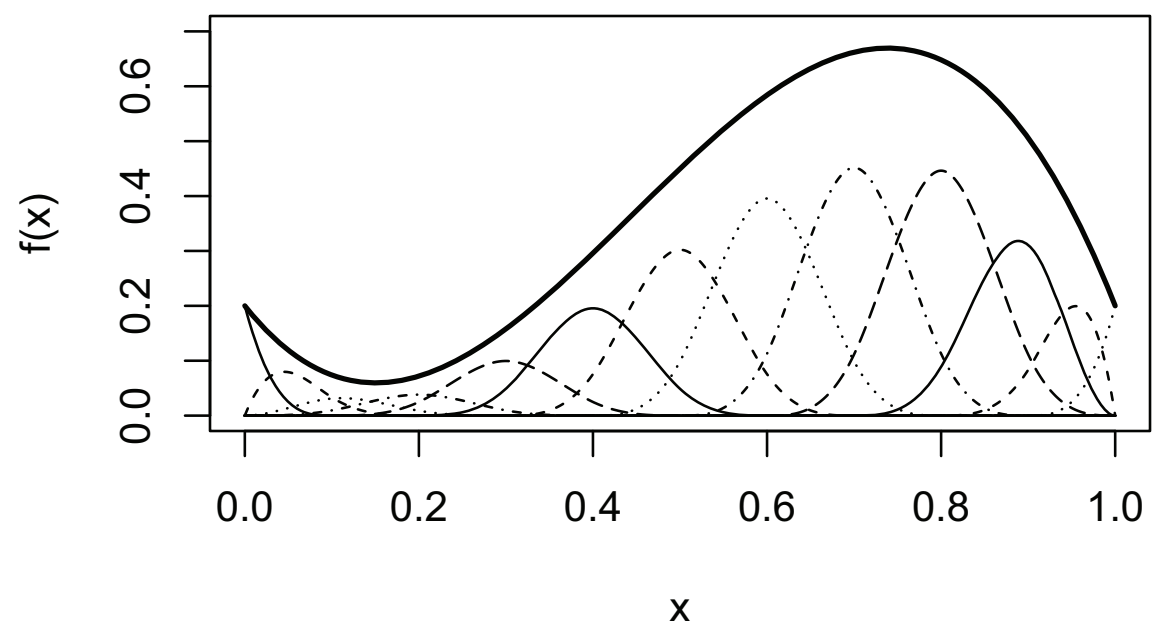

Figure 3: Example of a B-Spline and its basis functions multiplied by their respective coefficient values.

\subsection{Penalized Splines and Smoothing}

One of the many advantages of B-Splines is their great flexibility, however this is not always a desired quality. For instance, a spline that is too flexible may increase the variability of the parameter estimates. Likewise, noisy data might also produce unweildy estimates. These problems may be palliated by introduce smoothing mechanisms. For B-Splines, smoothing may be implemented by: a) reducing the order of the spline, b) reducing the number and changing the location of the knots, or c) using penalized methods.

\subsubsection{Lower Order Splines}

Reducing the order of a spline is equivalent to reducing the degree of the approximating polynomial on the the local intervals. In other words, this technique introduces smoothing by limiting the model's ability to pick up features in the data. Much like 
trying to fit a third degree polynomial with a straight line, reducing the order of a spline is a fairly crude smoothing method (Ramsay and Silverman, 2005).

\subsubsection{Modifing the Number and Location of Knots}

Not unlike reducing the order of the spline, modifying the number and location of knots achieves smoothing by modulating the spline's flexibility. The more knots there are within a fixed interval, the more flexible the spline becomes on that interval. In addition, the proximity of the knots has a significant impact on the shape of the spline. Knots that are closer together, to the extent where there are several at the same point, result in potentially sharper curves and even discontinuities.

Functions with areas of high and low curvature may be more accurately represented by a spline if its knots are respectively closer together or farther apart. Several computationally intensive adaptive procedures exist for determining the number and location of knots, however these techniques are not investigated in this thesis. Interested readers are referred to de Boor (1978), Hastie et al. (2009) and Ramsay and Silverman (2005).

\subsubsection{Regularization}

The third and more widely used method for smoothing splines is to apply a penalty to the loss function, so that one can use a large number of knots to avoid the issue of position and placement of the knots. The penalty is typically a loss function in some normed space, multiplied by a smoothing parameter, $\lambda$, which takes on values between zero and infinity (see Ramsay and Silverman, 2005; Ramsay et al., 2009;

Hastie et al., 2009, Chap. 5). When the smoothing parameter is zero we obtain the usual least squares fit, and when it nears infinity the penalty dominates the fitting of 
the coefficients. This type of spline is generally referred to as a penalized or smoothing spline.

For a given value of the smoothing parameter, $\lambda$, it is possible to obtain estimates for the smoothing spline coefficients by minimizing a penalized loss function. For the $L_{2}$ loss function, this amounts to minimizing the penalized residual squared sum, for which the general form is:

$$
p R S S=[\vec{y}-f(\vec{x})]^{\top} \boldsymbol{W}[\vec{y}-f(\vec{x})]+\lambda \int g(t) d t
$$

where $\vec{y}=\left\{y_{1}, y_{2}, \ldots, y_{n}\right\}, f(\vec{x})=\left\{f\left(x_{1}\right), f\left(x_{2}\right), \ldots, f\left(x_{n}\right)\right\} \boldsymbol{W}$ is a weight matrix (often the identity matrix), and $f(x)$ and $g(t)$ are respectively the function of interest and the penalty function.

Different penalties serve different purposes. For instance, if we wish to control the level of curvature of a spline, we could impose a penalty on the second derivative. Under the $L_{2}$ loss function, this involves integrating the square of the second derivative over the domain of the function. As usual, the amount of smoothing applied is determined by the smoothing parameter, $\lambda$. If the parameter is zero, we obtain the least squares fit. However, if $\lambda$ tends towards infinity, we obtain a linear fit (Ramsay and Silverman, 2005). For a fixed value of $\lambda$, estimates of the coefficients for BSplines penalized in this manner are again obtained by minimizing the penalized residual squared sum:

$$
p R S S=[\vec{y}-f(\vec{x})]^{\top} \boldsymbol{W}[\vec{y}-f(\vec{x})]+\lambda \int\left[f^{\prime \prime}(t)\right]^{2} d t
$$




\subsubsection{P-Splines}

Evaluating the integral of the square of the second derivative of a function can be done, but is a tedious computation. Eilers and Marx $(1996,2010)$ have developed another penalty based on the differences of neigbhouring coefficients of splines. These so called P-Splines, have penalties that are much easier to compute, and produce results very similar to those of B-Splines with penalties on their second derivatives. Basically, the approach uses finite differences to approximate the derivatives and sums of the integrals.

P-Splines use a large number of basis functions with equally spaced knots (much more than what is usually required to fit the function) and let their penalty regularize the coefficients. (Eilers and Marx, 1996) derive their P-Spline penalty from de Boor's equation for the derivatives of B-Splines.

Let $f(x)$ be as in (2.11), with $n$ equally spaced interior knots, and a $k+1$ order B-Spline basis as defined in (2.12). Supposing that $\Delta \alpha_{i}=\alpha_{i}-\alpha_{i-1}$ and that $\Delta t$ is the distance between knots, de Boor (1978)'s equation is given by:

$$
\begin{aligned}
\frac{d}{d x} f(x) & =\frac{d}{d x} \sum_{i=1}^{n+k+1} \alpha_{i} \cdot B_{i, k}(x) \\
& =\sum_{i=1}^{n+k+1} \alpha_{i} \cdot B_{i, k}^{\prime}(x) \\
& =\sum_{i=2}^{n+k+1} \frac{\alpha_{i}-\alpha_{i-1}}{\left(t_{i+k-1}-t_{i}\right) /(k-1)} B_{i, k-1}(x) \\
& =\sum_{i=2}^{n+k+1} \frac{\alpha_{i}-\alpha_{i-1}}{((k-1) \Delta t) /(k-1)} B_{i, k-1}(x) \\
& =\sum_{i=2}^{n+k+1} \frac{\alpha_{i}-\alpha_{i-1}}{\Delta t} B_{i, k-1}(x) .
\end{aligned}
$$


Upon rearranging the terms, Eilers and Marx (1996) obtained:

$$
\begin{aligned}
\Delta t \sum_{i=1}^{n+k+1} \alpha_{i} \cdot B_{i, k}^{\prime}(x) & =\sum_{i=2}^{n+k+1} \alpha_{i-1} \cdot B_{i, k-1}(x)-\sum_{i=2}^{n+k+1} \alpha_{i} \cdot B_{i+1, k-1}(x) \\
& =-\sum_{i=2}^{n+k+1} \Delta \alpha_{i} \cdot B_{i, k-1} .
\end{aligned}
$$

By induction, for $\Delta^{2} \alpha_{i}=\Delta \Delta \alpha_{i}=\alpha_{i}-2 \alpha_{i-1}+\alpha_{i-2}$, they also obtained:

$$
\Delta t^{2} \sum_{i=1}^{n+k+1} \alpha_{i} \cdot B_{i, k}^{\prime \prime}=\sum_{i=3}^{n+k+1} \Delta^{2} \alpha_{i} \cdot B_{i, k-2}
$$

which may be generalized to the $d^{\text {th }}$ order difference.

The above implies that it is possible to create smoothing spline penalties based on the differences of neighbouring coefficients that are roughly equivalent to those based on a spline's $d^{t h}$ derivative. Using the $L_{2}$ loss function, for $0 \leq d \leq k$, such a penalty would produce the following penalized sum of squared residuals:

$$
\begin{aligned}
p R S S & =[\vec{y}-f(\vec{x})]^{\top} \boldsymbol{W}[\vec{y}-f(\vec{x})]+\lambda \sum_{i=1+d}^{n+k+1}\left[\Delta^{d} \alpha_{i}\right]^{2} d t \\
& =[\vec{y}-f(\vec{x})]^{\top} \boldsymbol{W}[\vec{y}-f(\vec{x})]+\lambda \vec{\alpha} \boldsymbol{D}_{d}^{\top} \boldsymbol{D}_{d} \vec{\alpha} \\
& =[\vec{y}-\boldsymbol{B} \vec{\alpha}]^{\top} \boldsymbol{W}[\vec{y}-\boldsymbol{B} \vec{\alpha}]+\lambda \vec{\alpha} \boldsymbol{D}_{d}^{\top} \boldsymbol{D}_{d} \vec{\alpha},
\end{aligned}
$$

where $\boldsymbol{W}$ is a diagonal matrix of possible weights, $\boldsymbol{B}$ is the matrix of basis functions, $\lambda$ is the smoothing parameter, and $\boldsymbol{D}_{d}$ is the matrix representation of the $d^{\text {th }}$ difference operator $\Delta^{d}$ as in Eilers and Marx (1996). The least squares estimate for (2.13) may be obtained by solving: 


$$
\hat{\vec{\alpha}}=\left(\boldsymbol{B}^{\top} \boldsymbol{W} \boldsymbol{B}+\lambda \boldsymbol{D}_{d}^{\top} \boldsymbol{D}_{d}\right)^{-1} \boldsymbol{W} \boldsymbol{B}^{\top} \boldsymbol{y} .
$$

As suggested by the form of the least squares estimate, P-Splines are a special case of generalized ridge regression (Eilers and Marx, 2010, 1996). Particularly, when $d=0$, using the P-Spline penalty is equivalent to performing a ridge regression on the coefficients, since $\boldsymbol{D}_{0}=\boldsymbol{I}$.

P-Splines may also be used in general linear models (GLMs). In many cases, the penalty term (or a transformation thereof) is subtracted from the log-likelihood. For instance, if the link function is canonical, we obtain a penalized log-likelihood of the form:

$$
L=l(y ; \alpha)-\frac{\lambda}{2} \sum_{i=1+d}^{n+k+1}\left[\Delta^{d} \alpha_{i}\right]^{2} .
$$

Parameter estimates may then be obtained by maximizing (2.15) numerically.

\subsection{Selecting the Smoothing Parameter}

The main difficulty associated with regularization is not the fitting of the coefficients, but rather the selection of the smoothing parameter. Finding the optimal value of the smoothing paramter, $\lambda$, is not always straightforward since in most cases a closed form expression does not exsist. In addition, simply optimizing the penalized log-likelihood does not take into account the amount of smoothing being applied. If one tries to optimize $\lambda$ directly, it will always choose an overparameterized model. For example, if $\lambda=0$ the model will be fully saturated. For this reason, one needs to optimize $\lambda$ using a measure that also takes into account the number of effective parameters in the model. 
For parametric estimation, the number of effective parameters are predetermined by the choice of model, however when smoothing is involved the effective number of parameters is not quite as easy to determine. Consider the Hat matrix, defined as $\boldsymbol{H}=\boldsymbol{B}\left(\boldsymbol{B}^{\top} \boldsymbol{B}\right)^{-1} \boldsymbol{B}^{\top}$, which plays an important role for linear models. Specifically, it projects the data onto the parameter space and allows for the estimation of coefficients. The trace of the Hat matrix, $\operatorname{Tr}(\boldsymbol{H})$, is equal to the dimension of the parameter space, and hence the number of parameters. The smoothing equivalent ${ }^{2}$ of the Hat matrix in the case of penalized linear models is known as the Smoothing matrix and is defined as:

$$
\boldsymbol{S}_{\lambda}=\boldsymbol{B}\left(\boldsymbol{B}^{\top} \boldsymbol{B}+\lambda \boldsymbol{P}\right)^{-1} \boldsymbol{B}^{\top}
$$

where $\boldsymbol{B}$ is the design matrix, $\lambda$ is the smoothing parameter, and $\boldsymbol{P}$ is the penalty matrix (Hastie et al., 2009). Analogously, the trace of the smoothing matrix, $\operatorname{Tr}\left(\boldsymbol{S}_{\lambda}\right)$, is equal to the number of effective parameters in the model, which are often referred to as the effective degrees of freedom. Note that $\operatorname{Tr}\left(\boldsymbol{S}_{\lambda}\right)$ is not integer-valued, and decreases for increasing $t$.

There are many available measures that make use of the effective degrees of freedom to ascertain an optimal level smoothing. Among the measures most often encountered figure cross-validation $(\mathrm{CV})$, generalized cross-validation $(\mathrm{GCV})$, restricted maximum likelihood (REML), Akaike's information criterion (AIC), and the Bayesian information criterion (BIC) (Eilers and Marx, 1996; Hastie et al., 2009). We have chosen to use the Bayesian information criterion in this thesis as the model considered is not Gaussian, and because its components are a by-product of the estimation process.

\footnotetext{
${ }^{2}$ While both fulfill similar roles and are symmetric, positive, semidefinite matrices, the Hat matrix is idempotent and preserves the dimension of the parameter space, $\boldsymbol{H}^{\top} \boldsymbol{H}=\boldsymbol{H}$, whereas the Smoothing matrix reduces it, $\boldsymbol{S}_{\lambda}^{\top} \boldsymbol{S}_{\lambda} \leq \boldsymbol{S}_{\lambda}$ (Hastie et al., 2009).
} 
The BIC is defined as:

$$
B I C(\lambda)=-2 \cdot \log (L(\vec{\theta}))+\log (n) \times \operatorname{Tr}\left(\boldsymbol{S}_{\lambda}\right)
$$

where $n$ is the number of data points, $\operatorname{Tr}\left(\boldsymbol{S}_{\lambda}\right)$ is the number of effective degrees of freedom (as defined above), and $L(\vec{\theta})$ is the log-likelihood, which is functionally dependent on the parameter vector $\vec{\theta}$ (Hastie et al., 2009).

Optimizing a measure, such as the BIC, that takes into account the log-likelihood and the effective degrees of freedom will yield a value for the smoothing parameter that optimizes the bias-variance trade-off, while taking into account the complexity of the model. 


\section{Chapter 3}

\section{Model}

The present section expands the results found in Kalbfleisch and Lawless's The Analysis of Panel Data Under a Markov Assumption (1985). Panel data modeled under a Markov assumption, as proposed by these authors, analyzes the transition intensities of a time dependent process by means of a series of constant parameters. The proposed method aims to modify this technique through the use of splines and penalized splines, and is contrasted with Kalbfleisch and Lawless's (1985) approach when appropriate. Beginning with spline notation, we will cover transition probabilities and intensities, maximum likelihood estimation, derived values, as well as possible extensions.

\subsection{Spline Notation}

Recall that splines are generally of the form presented in (2.11). For convenience, we will alter this notation slightly. Let:

$$
g_{u}(x)=\sum_{v=1}^{b_{u}} \psi_{(u, v)} B_{(u, v)}(x) \text { for } u=1, \ldots, b,
$$

where the $\psi_{(u, v)}$ 's are the coefficients of the basis functions, which are denoted by the $B_{(u, v)}$ 's, $b$ is the number of independent splines in the model, and $b_{u}$ is the number of 
coefficients of the $u^{\text {th }}$ spline.

Among the myriad choices of basis, we will use the B-Spline basis described by de Boor in A Practical Guide to Splines (1978), whose properties are briefly summarized in Section 2.4. The selection of the B-Spline basis is justified by its interesting numerical properties and readily available software ${ }^{1}$.

As we will see in Section 3.3, there is a considerable advantage to optimizing the target function using a one-to-one exponential transformation. Although this transformation may introduce certain numerical difficulties ${ }^{2}$, it allows us to perform an unconstrained optimization on an otherwise constrained parameter space. Applying an exponential transformation to (3.1) produces:

$$
f_{u}(x)=\exp \left\{g_{u}(x)\right\}=\exp \left\{\sum_{v=1}^{b_{u}} \psi_{(u, v)} B_{(u, v)}(x)\right\} \text { for } u=1, \ldots, b .
$$

Despite the fact that the $\psi_{(u, v)}$ 's have a double subscript which identifies the spline to which each parameter is associated, they form a vector, $\vec{\Psi}=\left\{\psi_{(u, v)}\right\}_{b_{u v} \times 1}$, of length $b_{u v}=\sum_{u=1}^{b} b_{u}$.

\footnotetext{
${ }^{1}$ We have implmented the proposed technique using the Spline package in R (R Core Team, 2013). There are, however, several other programs capable of handling splines.

${ }^{2}$ The exponential transformation does not eliminate the challenges inherent with estimating values near the boundaries of the parameter space. In the case of strictly positive numbers, estimates near zero and infinity will cause considerable problems.
} 


\subsection{Transition Probabilities}

Recall from Section 2 that panel data may be analyzed as a continuous-time stochastic process, under a Markov assumption. Under these conditions, panel studies suppose that a sample of individuals move independently among $k$ exhaustive and mutually exclusive states that make up the space $\mathcal{S}=\{1,2, \ldots, k\}$. The sampled individuals are observed at a finite number of discrete times over a given period, say: $t_{0} \leq t_{1} \leq \ldots \leq t_{m}$. Using the count data for each pair of adjacent observation times, called panels, we construct a series of $k \times k$ matrices.

For each panel, there is a corresponding $k \times k$ transition probablity matrix, $\boldsymbol{P}(s, t)$. Letting $X(t)$ be the state occupied by any given individual at time $t$, the transition probabilities, $p_{i j}(s, t)$ 's over the interval $[s, t]$ are defined by $(2.1)$ :

$$
p_{i j}(s, t)=P\{X(t)=j \mid X(s)=i\}
$$

for $i, j=1, \ldots, k$ and $t_{0} \leq s \leq t \leq t_{m}$, such that $\sum_{j=1}^{k} p_{i j}(s, t)=1$.

Recall that transition probabilities may be time-homogeneous or non-homogeneous. Kalbfleisch and Lawless are primarily concerned with the time-homogeneous case, for which the transition probabilities only depend upon the elapsed time, $t-s$ :

$$
\boldsymbol{P}(s, t)=\boldsymbol{P}(0, t-s)
$$

We consider the non-homogeneous case, for which the initial and final time points, respectively $s$ and $t$, are relevant. 


\subsection{Transition intensities}

The probability transition matrix, $\boldsymbol{P}(s, t)$, may be expressed in the form of transition intensities. The transition intensities for the non time-homogeneous case are defined by (2.7), and may be simplified the time-homogeneous case. For convenience, we consider the matrix $\boldsymbol{Q}(s, t)$, defined in (2.6), which is the integrated intensity matrix over the interval $[s, t]$. For both the homogeneous and non-homogeneous cases, the integrated intensity matrix $\boldsymbol{Q}(s, t)$ has null row sums and positive, functionally independent ${ }^{3}$, continuous off-diagonal elements.

Combining the integrated intensity functions in (2.6) with the splines defined in (3.2), we obtain:

$$
q_{i j}(s, t, \vec{\Psi})= \begin{cases}\int_{s}^{t} \exp \left\{\sum_{v=1}^{b_{u}} \psi_{(u, v)} B_{(u, v)}(x)\right\} d x & \text { for } i \neq j \\ -\sum_{j \neq i} q_{i j}(s, t, \vec{\Psi}) & \text { for } i=j,\end{cases}
$$

where $q_{i j}(s, t, \vec{\Psi})$ is associated with the $u^{t h}$ spline. Note that the integrated intensity matrix, $\boldsymbol{Q}(s, t, \vec{\Psi})$, is now a function of the coefficient vector, $\vec{\Psi}$.

The elements of $\boldsymbol{Q}(s, t)$ admit first derivatives with respect to $\vec{\Psi}$. Taking the first partial derivative of (3.3) with respect to the $\psi_{\tau_{1}}$, where $\tau_{1} \in\left(u_{1}, v\right)$, yields:

\footnotetext{
${ }^{3} q_{i j}(s, t)$ and $q_{i^{\prime} j^{\prime}}(s, t)$ are functionally independent if they do not depend on the same parameters.
} 


$$
\frac{\partial q_{i j}(s, t, \vec{\Psi})}{\partial \psi_{\tau_{1}}}= \begin{cases}\int_{s}^{t} \exp \left\{\sum_{v=1}^{b_{u}} \psi_{(u, v)} B_{(u, v)}(x)\right\} B_{\tau_{1}}(x) d x & \text { for } i \neq j, u=u_{1} \\ -\sum_{j \neq i} \frac{\partial q_{i j}(s, t, \vec{\Psi})}{\partial \psi_{\tau_{1}}} & \text { for } i=j, u=u_{1} \\ 0 & \text { for } u \neq u_{1},\end{cases}
$$

Similarly, the second partial derivatives of $\boldsymbol{Q}(s, t, \vec{\Psi})$ with respect to $\psi_{\tau_{1}}$ and $\psi_{\tau_{2}}$, for $\tau_{1} \in\left(u_{1}, v\right)$ and $\tau_{2} \in\left(u_{2}, v\right)$ are:

$$
\frac{\partial^{2} q_{i j}(s, t, \vec{\Psi})}{\partial \psi_{\tau_{1}} \partial \psi_{\tau_{2}}}= \begin{cases}\int_{s}^{t} \exp \left\{\sum_{v=1}^{b_{u}} \psi_{(u, v)} B_{(u, v)}(x)\right\} B_{\tau_{1}}(x) B_{\tau_{2}}(x) d x & \text { for } i \neq j, u=u_{1}=u_{2} \\ -\sum_{j \neq i} \frac{\partial^{2} q_{i j}(s, t, \vec{\Psi})}{\partial \psi_{\tau_{1}} \partial \psi_{\tau_{2}}} & \text { for } i=j, u=u_{1}=u_{2} \\ 0 & \text { for } u \neq u_{1}, u \neq u_{2}, u_{1} \neq u_{2} .\end{cases}
$$

Note that the first and second derivatives conserve the null row sum condition. These conditions may be expressed as: $\sum_{j} q_{i j}(s, t, \vec{\Psi})=0, \sum_{j} \frac{\partial q_{i j}(s, t, \vec{\Psi})}{\partial \tau_{1}}=0, \sum_{j} \frac{\partial^{2} q_{i j}(s, t, \vec{\Psi})}{\partial \tau_{1} \partial \tau_{2}}=0$.

\subsection{Expressing Transition Probabilities and Inten- sities}

Updating (2.9) to reflect the dependence of (3.3) on $\vec{\Psi}$, we obtain the relation:

$$
\boldsymbol{P}(s, t, \vec{\Psi})=\exp \{\boldsymbol{Q}(s, t, \vec{\Psi})\}
$$


For given values of $\vec{\Psi}$, the elements of the previous equation are constant, so it is possible to compute $\boldsymbol{P}(s, t \mid \vec{\Psi})$ by means of the canonical decomposition described in (2.10) (Kalbfleisch and Lawless, 1985). Letting $\boldsymbol{Q}(s, t \mid \vec{\Psi})=\boldsymbol{A} \boldsymbol{D} \boldsymbol{A}^{-1}$, and $\boldsymbol{D}=$ $\operatorname{diag}\left(d_{1}(s, t), d_{2}(s, t), \ldots, d_{k}(s, t)\right)$, where the $d_{i}(s, t)$ are the eigenvalues of $\boldsymbol{Q}(s, t \mid \vec{\Psi})$ and $\boldsymbol{A}$ is the matrix of corresponding eigenvectors, we obtain:

$$
\boldsymbol{P}(s, t \mid \vec{\Psi})=\boldsymbol{A} \operatorname{diag}\left(e^{d_{1}(s, t)}, e^{d_{2}(s, t)}, \ldots, e^{d_{k}(s, t)}\right) \boldsymbol{A}^{-1}
$$

Even if $\boldsymbol{P}(s, t, \vec{\Psi})$ is dependent on $\vec{\Psi}$, the functions that link the two are rather complex and do not lend themselves well to differentiation. However, it is possible to exploit the canonical decomposition of $\boldsymbol{Q}(s, t \mid \vec{\Psi})$ to obtain the derivatives of $\boldsymbol{P}(s, t \mid \vec{\Psi})$ (Kalbfleisch and Lawless, 1985). Let $\boldsymbol{G}^{(\tau)}$ be a function of the eigenvectors and the first derivatives of $\boldsymbol{Q}(s, t \mid \vec{\Psi})$ with respect to $\psi_{\tau} \in \vec{\Psi}$, such that:

$$
\boldsymbol{G}^{(\tau)}=\boldsymbol{A}^{-1}\left(\frac{\partial \boldsymbol{Q}(s, t \mid \vec{\Psi})}{\partial \psi_{\tau}}\right) \boldsymbol{A}=\left\{g_{i j}^{(\tau)}\right\}_{k \times k}
$$

$\boldsymbol{G}^{(\tau)}$ is then used to construct $\boldsymbol{V}_{\tau}=\left\{v_{i j}^{(\tau)}\right\}_{k \times k}$, such that:

$$
v_{i j}^{(\tau)}= \begin{cases}g_{i j}^{(\tau)} \frac{e^{d_{i}(s, t)}-e^{d_{j}(s, t)}}{\left(\frac{d_{i}(s, t)-d_{j}(s, t)}{t-s}\right)} & \text { for } i \neq j \\ g_{i j}^{(\tau)}(s, t) e^{d_{i}(s, t)} & \text { for } i=j .\end{cases}
$$

Finally, we obtain the derivative of $\boldsymbol{P}(s, t \mid \vec{\Psi})$ with respect to $\psi_{\tau}$ :

$$
\frac{\partial \boldsymbol{P}(s, t \mid \vec{\Psi})}{\partial \psi_{\tau}}=\boldsymbol{A} \boldsymbol{V}_{\tau} \boldsymbol{A}^{-1} \text { for } \tau=1, \ldots, b_{u v}
$$

Using (3.5) and (3.6)-(3.8), it is possible to compute $\boldsymbol{P}(s, t \mid \vec{\Psi})$ and its derivatives without explicitly representing the transition probabilities as functions of $\vec{\Psi}$. 
Recall that $\boldsymbol{P}(s, t)$ is defined with conditions on its row sums, specifically: $\sum_{j=1}^{k} p_{i j}(s, t)=1$. This ensures that transitionning from a state into any other possible state sums to one, as required. Similarly, $\boldsymbol{Q}(s, t)$ has the row conditions $\sum_{j=1}^{k} q_{i j}(s, t)=0$. By construction, the conditions on $\boldsymbol{P}(s, t)$ and $\boldsymbol{Q}(s, t)$ are linked through (3.4). As discussed in Section 3.3, the derivatives of $\boldsymbol{Q}(s, t)$ conserve the null row sum condition. By extension, the derivatives of $\boldsymbol{P}(s, t)$ also respect the conditions on the row sums.

\subsection{Maximum Likelihood Estimation}

Using the representation developed in the previous sections, and letting $n_{i j l}$ denote the number of individuals in state $i$ at the time $t_{l-1}$ and in state $j$ at $t_{l}$, for $0 \leq t_{l-1} \leq$ $t_{l} \leq t_{m}$, we are able to construct the likelihood function. Conditioning on the number of individuals in each state at $t_{0}$, we obtain (Kalbfleisch and Lawless, 1985):

$$
L(\vec{\Psi})=\prod_{l=1}^{m}\left\{\prod_{i, j=1}^{k} p_{i j}\left(t_{l-1}, t_{l}, \vec{\Psi}\right)^{n_{i j l}}\right\} .
$$

For notational convenience, we will suppress the dependence of the probability transition matrix on the coefficient vector and let $\boldsymbol{P}\left(t_{l-1}, t_{l}, \vec{\Psi}\right)=\boldsymbol{P}\left(w_{l}\right)$, where $w_{l}=$ $\left(t_{l-1}, t_{l}\right)$ for $l=1, \ldots, m$. Consequently, the log-likelihood may be written as:

$$
\log (L(\vec{\Psi}))=\sum_{l=1}^{m} \sum_{i, j=1}^{k} n_{i j l} \cdot \log \left(p_{i j}\left(w_{l}\right)\right)
$$

Despite that the log-likelihood is not represented as an explicit function of $\vec{\Psi}$ it is

still possible to obtain the maximum likelihood estimate, $\hat{\vec{\Psi}}$, since the derivatives of the transition probabilities with respect to $\psi_{\tau}$ may be computed using (3.6)-(3.8). Hence, we may compute the elements of the gradient vector using the first partial 
derivatives (Kalbfleisch and Lawless, 1985):

$$
S_{\tau}(\vec{\Psi})=\frac{\partial \log (L(\vec{\Psi}))}{\partial \psi_{\tau}}=\sum_{l=1}^{m} \sum_{i, j=1}^{k} n_{i j l} \frac{\partial p_{i j}\left(w_{l}\right) / \partial \psi_{\tau}}{p_{i j}\left(w_{l}\right)}
$$

for $\tau=1, \ldots, b_{u v}$. The second partial derivatives are computed in a similar manner for $\tau_{1}, \tau_{2}=1, \ldots, b_{u v}$ :

$$
\frac{\partial^{2} \log (L(\vec{\Psi}))}{\partial \psi_{\tau_{1}} \partial \psi_{\tau_{2}}}=\sum_{l=1}^{m} \sum_{i, j=1}^{k} n_{i j l}\left\{\frac{\frac{\partial^{2} p_{i j}\left(w_{l}\right)}{\partial \psi_{\tau_{1}} \partial \psi_{\tau_{2}}}}{p_{i j}\left(w_{l}\right)}-\frac{\frac{\partial p_{i j}\left(w_{l}\right)}{\partial \psi_{\tau_{1}}} \cdot \frac{\partial p_{i j}\left(w_{l}\right)}{\partial \psi_{\tau_{2}}}}{p_{i j}^{2}\left(w_{l}\right)}\right\} .
$$

Using the fact that $\sum_{j=1}^{k} \frac{\partial^{2} p_{i j}\left(w_{l}\right)}{\partial \psi_{\tau_{1}} \partial \psi_{\tau_{2}}}=0$, and that $N_{i}\left(t_{l-1}\right) \times p_{i j}\left(w_{l}\right)=n_{i j l}$, where $N_{i}\left(t_{l-1}\right)=\sum_{j=1}^{k} n_{i j l}$ is the number of individuals in state $i$ at time $t_{l-1}$, we may take the expectation of the $n_{i j l}$ 's and rearrange the terms in (3.12) to obtain the Fisher information:

$$
E\left[-\frac{\partial^{2} \log (L(\vec{\Psi}))}{\partial \psi_{\tau_{1}} \partial \psi_{\tau_{2}}}\right]=\sum_{l=1}^{m} \sum_{i, j=1}^{k} \frac{E\left\{N_{i}\left(t_{l-1}\right)\right\}}{p_{i j}\left(w_{l}\right)} \frac{\partial p_{i j}\left(w_{l}\right)}{\partial \psi_{\tau_{1}}} \frac{\partial p_{i j}\left(w_{l}\right)}{\partial \psi_{\tau_{2}}}
$$

To avoid computing $E\left\{N_{i}\left(t_{l-1}\right)\right\}$, we use their observed values, as in Kalbfleisch and Lawless (1985),for $\tau_{1}, \tau_{2}=1, \ldots, b_{u v}$ :

$$
M_{\tau_{1} \tau_{2}}(\vec{\Psi})=\sum_{l=1}^{m} \sum_{i, j=1}^{k} \frac{N_{i}\left(t_{l-1}\right)}{p_{i j}\left(w_{l}\right)} \frac{\partial p_{i j}\left(w_{l}\right)}{\partial \theta_{\tau_{1}}} \frac{\partial p_{i j}\left(w_{l}\right)}{\partial \theta_{\tau_{2}}}
$$

Newton's method is used with $\boldsymbol{M}(\vec{\Psi})=\left\{M_{\tau_{1} \tau_{2}}(\vec{\Psi})\right\}_{b_{u v} \times b_{u v}}$, given the observed information, and the vector $\vec{S}(\vec{\Psi})=\left\{S_{\tau}(\vec{\Psi})\right\}_{b_{u v} \times 1}$. The update step for $\vec{\Psi}$ is then:

$$
\vec{\Psi}_{1}=\vec{\Psi}_{0}+M^{-1}\left(\vec{\Psi}_{0}\right) \vec{S}\left(\vec{\Psi}_{0}\right)
$$

where $\vec{\Psi}_{0}$ is the current value of $\vec{\Psi}$. This process is repeated until it converges to the 
maximum likelihood estimate, $\hat{\vec{\Psi}}$.

\subsection{Penalized Maximum Likelihood Estimation}

It is possible to introduce smoothing techniques to the maximum likelihood estimation process by applying some of the results found in Sections 2.5 and 2.6. Particularly, Eilers and Marx's P-Splines may be implemented by adding a penalty term to the loglikelihood given in (3.10). Adding such a penalty produces a penalized log-likelihood which may be written as:

$$
\log (L(\vec{\Psi}))=\sum_{l=1}^{m} \sum_{i, j=1}^{k} n_{i j l} \cdot \log \left(p_{i j}\left(w_{l}\right)\right)-\frac{\lambda}{2} \vec{\Psi}^{\top} \boldsymbol{R} \vec{\Psi}
$$

where $\lambda$ is the smoothing parameter and $\boldsymbol{R}$ is the $b_{u v} \times b_{u v}$ penalty matrix, which is block diagonal with block elements $\boldsymbol{D}_{d, u}^{\top} \boldsymbol{D}_{d, u}$. In this case $\boldsymbol{D}_{d, u}$ is the $d^{\text {th }}$ difference matrix, as in (2.13) and (2.14), associated with the $u^{\text {th }}$ function. If no smoothing is to be applied to the $u^{t h}$ function, $\boldsymbol{D}_{d, u}$ is replaced with a null matrix of corresponding dimensions. Consequently, the gradient is:

$$
\begin{aligned}
\vec{S}_{\lambda}(\vec{\Psi})_{b_{u v} \times 1} & =\left\{S_{\tau}(\vec{\Psi})\right\}_{b_{u v} \times 1} \\
& =\left\{\frac{\partial \log (L(\vec{\Psi}))}{\partial \psi_{\tau}}\right\}_{b_{u v} \times 1} \\
& =\left\{\sum_{l=1}^{m} \sum_{i, j=1}^{k} n_{i j l} \frac{\partial p_{i j}\left(w_{l}\right) / \partial \psi_{\tau}}{p_{i j}\left(w_{l}\right)}\right\}_{b_{u v} \times 1}-\lambda \boldsymbol{R} \vec{\Psi}
\end{aligned}
$$

for $\tau=1, \ldots, b_{u v}$. Similarly, the Hessian is: 


$$
\begin{aligned}
\boldsymbol{M}_{\lambda}(\vec{\Psi})_{b_{u v} \times b_{u v}} & =\left\{M_{\tau_{1} \tau_{2}}(\vec{\Psi})\right\}_{b_{u v} \times b_{u v}} \\
& =\left\{\sum_{l=1}^{m} \sum_{i, j=1}^{k} \frac{N_{i}\left(t_{l-1}\right)}{p_{i j}\left(w_{l}\right)} \frac{\partial p_{i j}\left(w_{l}\right)}{\partial \theta_{\tau_{1}}} \frac{\partial p_{i j}\left(w_{l}\right)}{\partial \theta_{\tau_{2}}}\right\}_{b_{u v} \times b_{u v}}-\lambda \boldsymbol{R},
\end{aligned}
$$

for $\tau_{1}, \tau_{2}=1, \ldots, \sum_{u=1}^{b} b_{u}$. Like in Section 3.5, the update step for $\vec{\Psi}$ is given by:

$$
\vec{\Psi}_{1}=\vec{\Psi}_{0}+M_{\lambda}^{-1}\left(\vec{\Psi}_{0}\right) \vec{S}_{\lambda}\left(\vec{\Psi}_{0}\right)
$$

where $\vec{\Psi}_{0}$ is the current estimate of $\vec{\Psi}$. For a given $\lambda$, this process is repeated until the estimate converges to the penalized maximum likelihood estimate, $\hat{\vec{\Psi}}_{\lambda}$. This value is then optimized with regards to a smoothing criterion, such as the Bayesian information criterion (BIC) discussed in Section 2.6.

\subsection{Derived Quantities}

Although the fitting procedure estimates model parameters and their variances, often times the researcher's interest is not in the parameters themselves, but in derived quantities and their variances. Usually, the functions of the desired quantities, say $f(\theta)$, are estimated by replacing their parameters, $\theta$, with their estimated values, $\hat{\theta}$. The variances of these functions are then estimated via the delta method. The delta method uses a Taylor series expansion of $f(\theta)$ around $\hat{\theta}$, and computes the vector of first derivatives, $\overrightarrow{\nabla f}(\hat{\theta})=\left\{\frac{\partial}{\partial \theta_{i}} f(\hat{\theta})\right\}$. Using the first derivatives and the variancecovariance matrix of the estimated parameters ${ }^{4}, \boldsymbol{V}(\hat{\theta})$, we construct the quadratic form:

\footnotetext{
${ }^{4}$ Note that variance-covariance matrix for the models proposed in Sections 3.5 and 3.6 are respectively $\boldsymbol{M}^{-1}(\hat{\vec{\Psi}})$ and $\boldsymbol{M}_{\lambda}^{-1}\left(\hat{\vec{\Psi}}_{\lambda}\right)$.
} 


$$
\hat{\operatorname{Var}}(f(\hat{\theta})) \approx \overrightarrow{\nabla f}(\hat{\theta})^{\top} \boldsymbol{V}(\hat{\theta}) \overrightarrow{\nabla f}(\hat{\theta})
$$

which provides an estimate of the variance of the desired quantity.

If there are several quantities to be estimated, $\vec{f}(x)=\left\{f_{1}(x), f_{2}(x), \ldots, f_{n}(x)\right\}$, the first derivatives may be grouped into a single matrix, $\boldsymbol{F}=\left\{\vec{\nabla} f_{i}(\hat{\theta})\right\}$. We then construct the quadratic form:

$$
\hat{\operatorname{Var}}(\vec{f}(\hat{\theta})) \approx \boldsymbol{F}(\hat{\theta})^{\top} \boldsymbol{V}(\hat{\theta}) \boldsymbol{F}(\hat{\theta})
$$

which produces an estimate of the variance-covariance matrix of the desired quantities.

\subsection{Extensions}

The techniques outlined in Sections 3.1-3.7 may be extended using a few simple modifications. For instance it is possible to use different basis functions, non-uniform knots, etc. It is also possible to introduce covariate values, redefine the operation time, as well as introduce random effects ${ }^{5}$.

\subsubsection{Covariates}

Introducing covariates may be accomplished using the proportional intensity approach employed by Kalbfleisch and Lawless (1985). Particularly, one or several indicator functions may be added to the intensity matrix, and thus the log-likelihood. Modifying (3.10) to include the covariate vector $\vec{z}_{h}=\left(z_{1 h}, z_{2 h}, \ldots, z_{s h}\right)$, we obtain:

\footnotetext{
${ }^{5}$ The current discussion does not cover the topic of random effects, although it is certainly a avenue to be explored in the future.
} 


$$
\log (L(\vec{\Psi}))=\sum_{h=1}^{r} \sum_{l=1}^{m} \sum_{i, j=1}^{k} n_{i j l}^{h} \cdot \log \left(p_{i j}\left(w_{l}, \vec{z}_{h}\right)\right)
$$

where $n_{i j l}^{h}$ are the number of individuals with the covariate values $\vec{z}_{h}$ that are in state $i$ at time $t_{l-1}$ and state $j$ at time $t_{l}$. Fitting the covariate model uses the same scoring procedure as the usual model except that (3.11) and (3.14) include a level for each covariate value (Kalbfleisch and Lawless, 1985):

$$
\begin{gathered}
S_{\tau}(\vec{\Psi})=\sum_{h=1}^{r} S_{\tau}^{(h)}(\vec{\Psi}), \\
M_{\tau_{1} \tau_{2}}(\vec{\Psi})=\sum_{h=1}^{r} M_{\tau_{1} \tau_{2}}^{(h)}(\vec{\Psi}) .
\end{gathered}
$$

If smoothing is being used, (3.16)-(3.18) must reflect the above, and the penalty matrix $\boldsymbol{R}$ must be adjusted appropriately.

\subsection{Embedability and Estimability}

There are certain situations that prove to be problematic for the estimation of parameters in Markov type models. In addition the scenarios where the parameters are inadmissible, or intractable, we find conditions under which there exist multiple intensity matrices $\boldsymbol{Q} \in \mathcal{Q}$ such that $\exp (\boldsymbol{Q})=\boldsymbol{P}$. This is known as the embedablility problem. Since the fitting procedures presented in Kalbfleisch and Lawless's article (1985), as well as in Sections 3.1-3.7 approximate the underlying probability distributions by modulating the intensity function coefficients within the parameter space of $\mathcal{Q}$, the conditions under which the algorithm will fail to produce an appropriate $\boldsymbol{P}$ are in large part circumvented. However, the problem that arises is that the algorithm may fail to converge to a specific $\boldsymbol{Q}$ based on the derivatives of $\boldsymbol{P}$. Additionally, 
there may not exist a $\boldsymbol{Q} \in \mathcal{Q}$ such that $\exp (\boldsymbol{Q})=\boldsymbol{P}$. Under these circumstances, the algorithm will identify a $\boldsymbol{P}$ close to the true values, which may result in very poor estimates (Kalbfleisch and Lawless, 1985). 


\section{Chapter 4}

\section{Results}

In order to demonstrate the techniques proposed in Section 3, we apply B-Spline and P-Spline based models to example and simulated data.

\subsection{Simulation}

Here we investigate the statistical properties of the proposed approach via a simulation study. The simulation study uses a three state model with three indpendent and arbitrary intensity functions for state 1 to state 2 (intensity 1 ), state 2 to state 1 (intensity 2), and state 1 to state 3 (intensity 3) transitions. The intensities have been chosen such that the expected number of transitions ${ }^{1}$ is 5 for non-absorbing states, and 1 for absorbing states. These functions are depicted by the blue lines in Figures 4-9 and 16-21.

In addition to the characteristics listed above, the simulation uses two levels for the sample sizes, as well as three levels for the number of panels (intervals between observation points). Consequently, the states occupied by the 200 or 500 individuals will be examined over 10, 25 or 50 panels. Observation times need not be evenly

\footnotetext{
${ }^{1}$ The expected number of transitions may be obtained by integrating the transition intensity over $t$.
} 
spaced, however for the purpose of this demonstration they have been set at equidistant points.

\subsubsection{B-Splines}

Applying the techniques outlined in Section 3 to fit a cubic B-Spline based model to the simulation data described above requires selecting the number and location of knots. This is a fairly complex numerical problem for which there exists several well known optimization procedures (de Boor, 1978; Hastie et al., 2009). Unfortunately, these procedures are rather involved, so for this simulation three knot locations have been retained according to best visual fit: $0.25,0.50,0.75$. Incidentally, these locations happen to conincide with the areas of high curvature of the most complex intensity function.

The results of the simulation, using 1000 generated data sets for each level, are presented in Figures 4-15. For the first six figures, the mean and median estimates are represented by the green and red lines respectivly, and a sample of fit values are shown in grey. From these figures, we observe that the spline estimates converge towards the true intensity functions, represented by the blue lines, as the sample size and number of panels increase. The number of panels has a particularly dramatic effect on the model's ability to properly estimate more complex functions. This is consistent with the idea that more frequent observations provide a better quality image of the features of the underlying process.

While splines are very flexible, they are limited by the positions of their knots, as well as the availability of data. Figures 4-9 reveal noticeable bias near the edges of the data ${ }^{2}$, as well as in areas of high curvature for the simulated data sets with few

\footnotetext{
${ }^{2}$ B-Splines, much like the polynomials that make up their basis, become unpredictable outside the range of the data. Unless assumptions are made about the behaviour of the model beyond this
} 

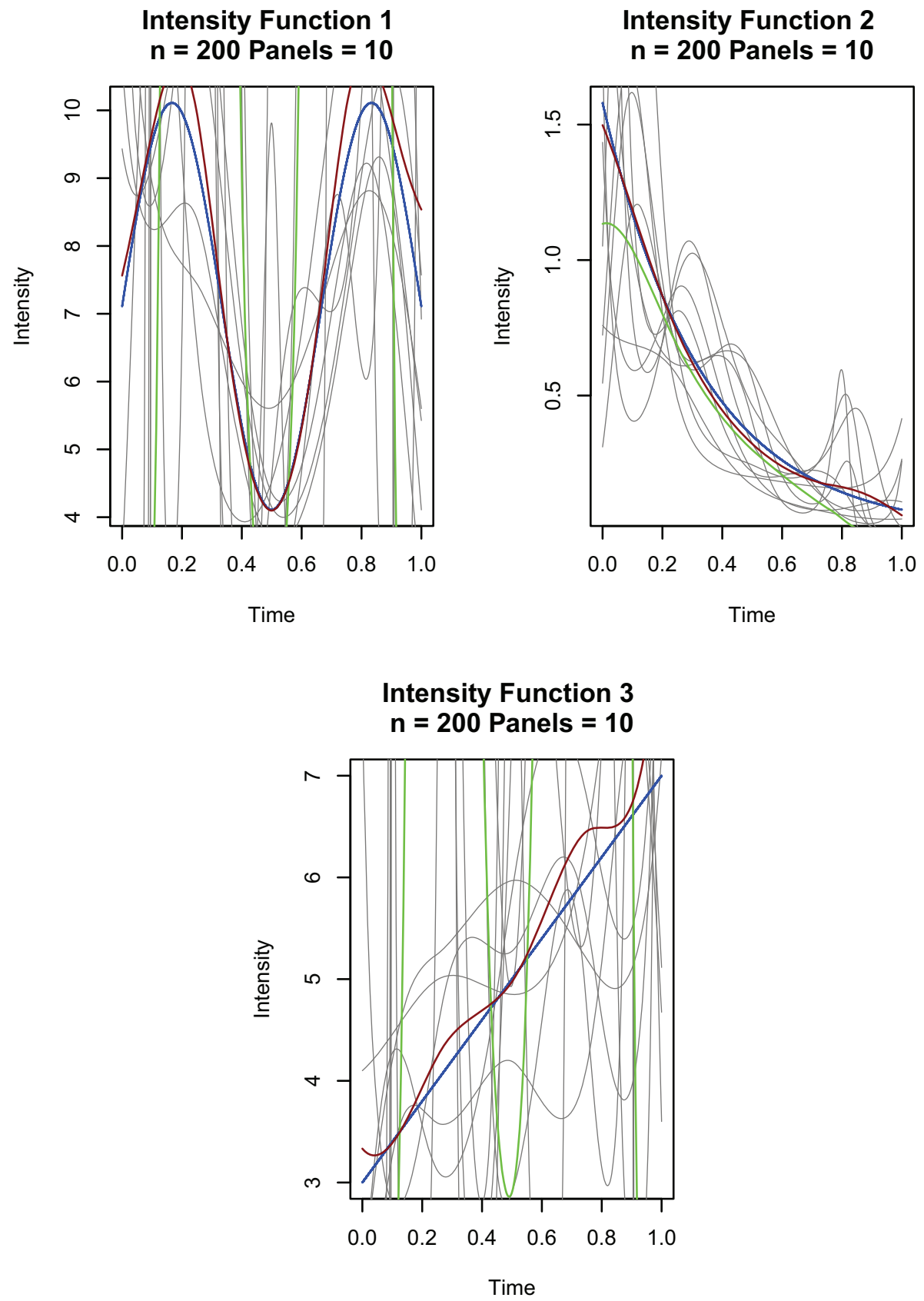

Figure 4: B-Spline based estimates of simulated data described in Section 4.1 with a cubic basis and knots set at $0.25,0.50$, and 0.75 . The true, mean and median values are respectively represented by the blue, green and red lines. A sample of fits are also show in grey. 

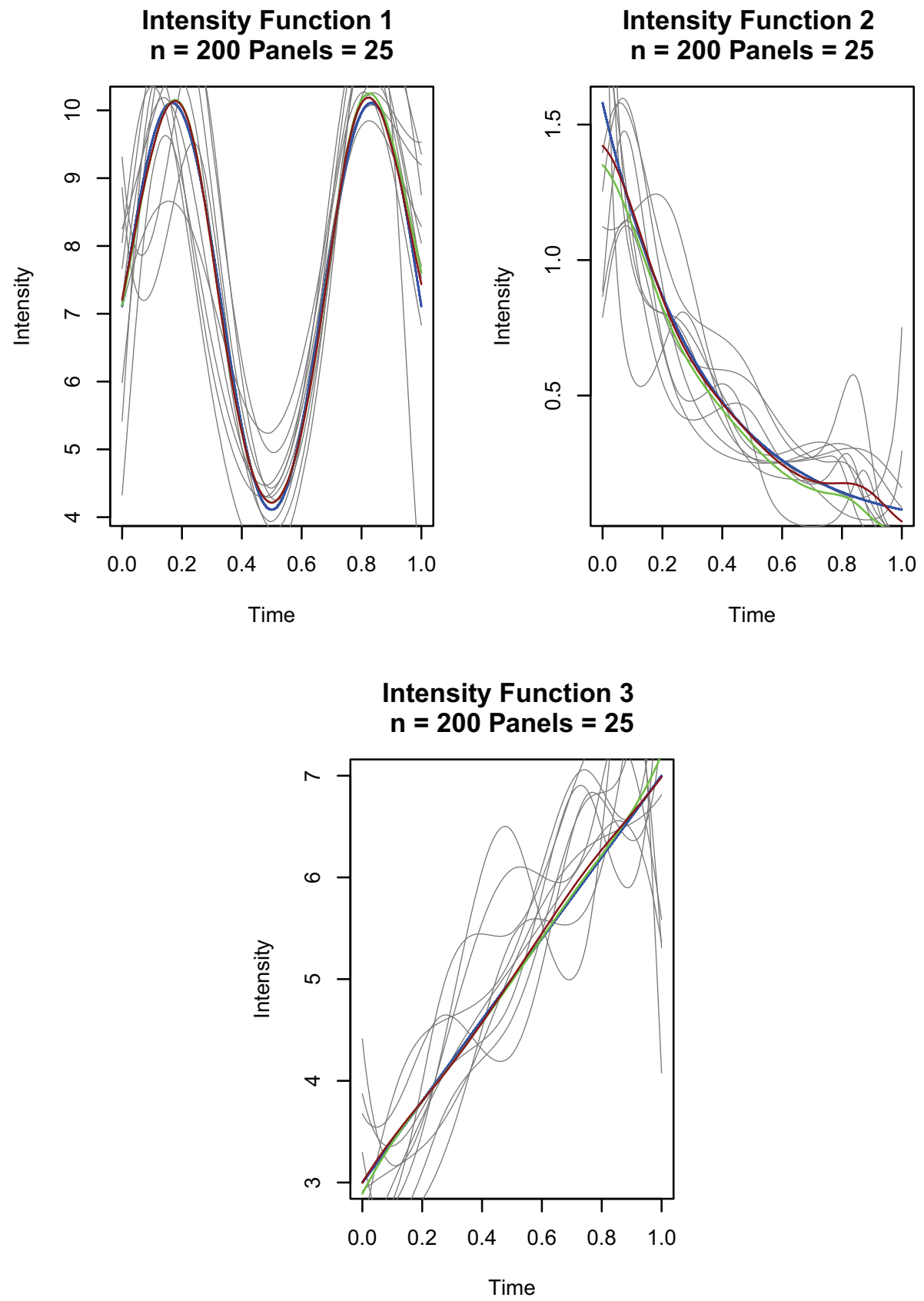

Figure 5: B-Spline based estimates of simulated data described in Section 4.1 with a cubic basis and knots set at $0.25,0.50$, and 0.75 . The true, mean and median values are respectively represented by the blue, green and red lines. A sample of fits are also show in grey. 

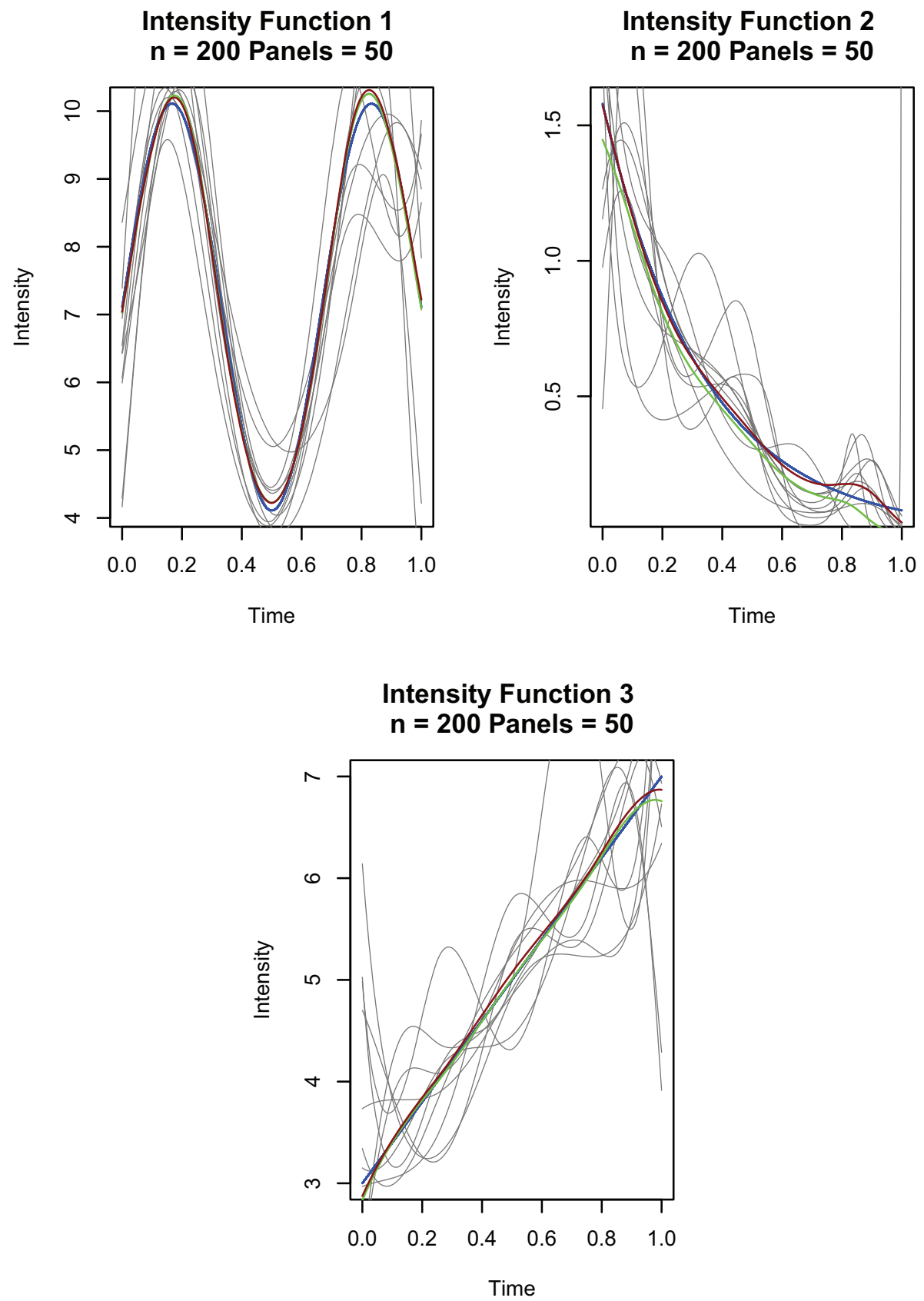

Figure 6: B-Spline based estimates of simulated data described in Section 4.1 with a cubic basis and knots set at $0.25,0.50$, and 0.75 . The true, mean and median values are respectively represented by the blue, green and red lines. A sample of fits are also show in grey. 

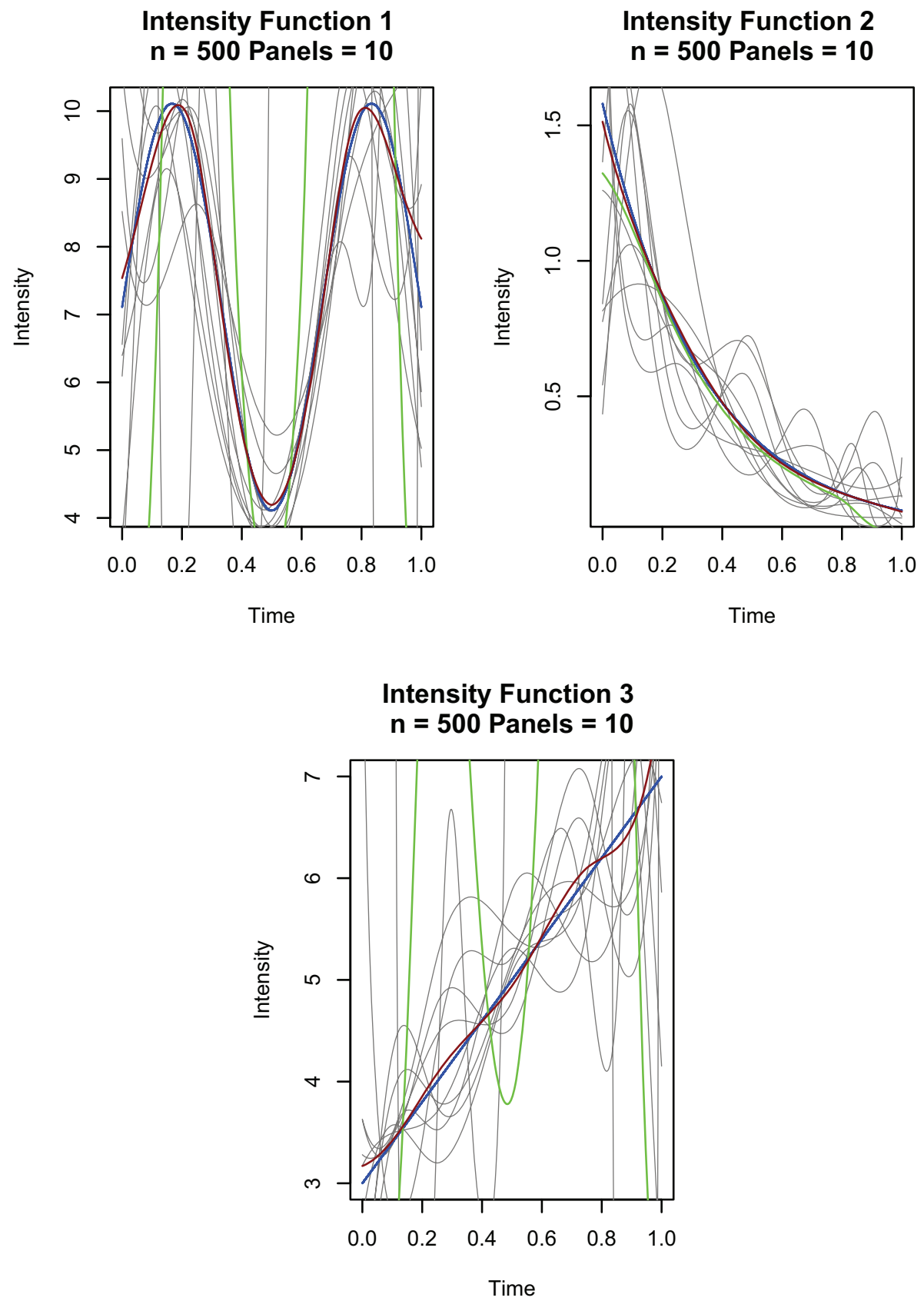

Figure 7: B-Spline based estimates of simulated data described in Section 4.1 with a cubic basis and knots set at $0.25,0.50$, and 0.75 . The true, mean and median values are respectively represented by the blue, green and red lines. A sample of fits are also show in grey. 

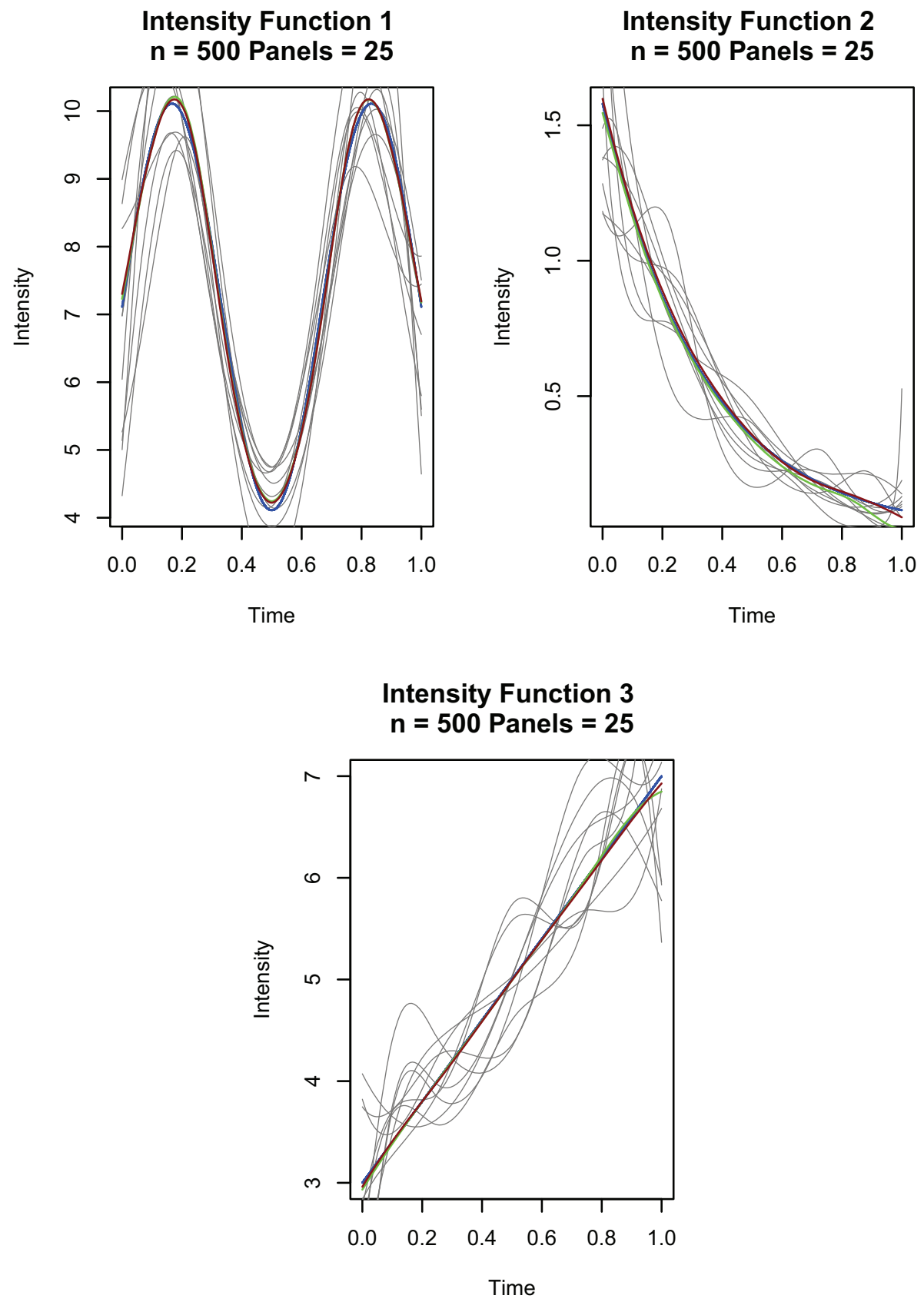

Figure 8: B-Spline based estimates of simulated data described in Section 4.1 with a cubic basis and knots set at $0.25,0.50$, and 0.75 . The true, mean and median values are respectively represented by the blue, green and red lines. A sample of fits are also show in grey. 

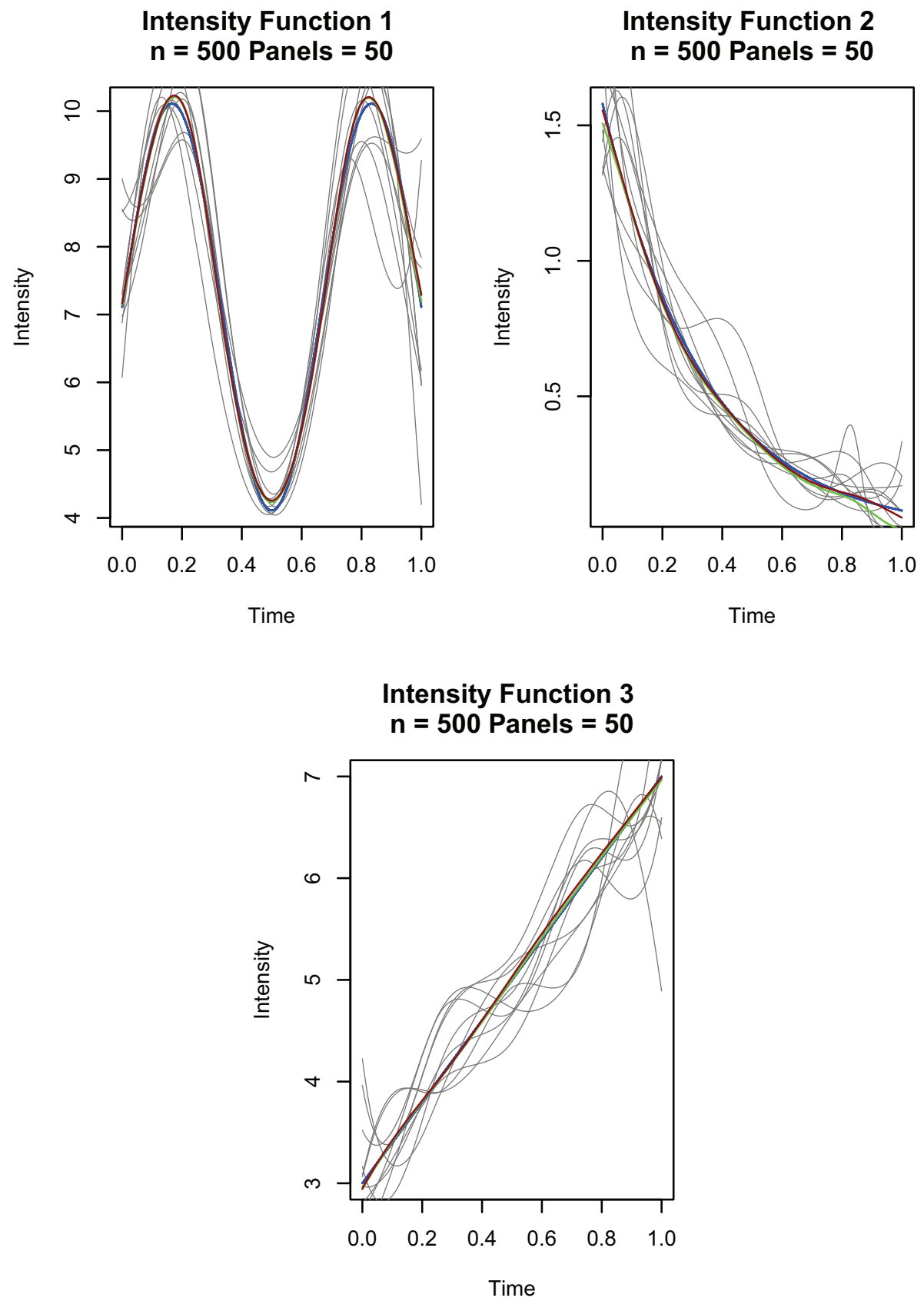

Figure 9: B-Spline based estimates of simulated data described in Section 4.1 with a cubic basis and knots set at $0.25,0.50$, and 0.75 . The true, mean and median values are respectively represented by the blue, green and red lines. A sample of fits are also show in grey. 

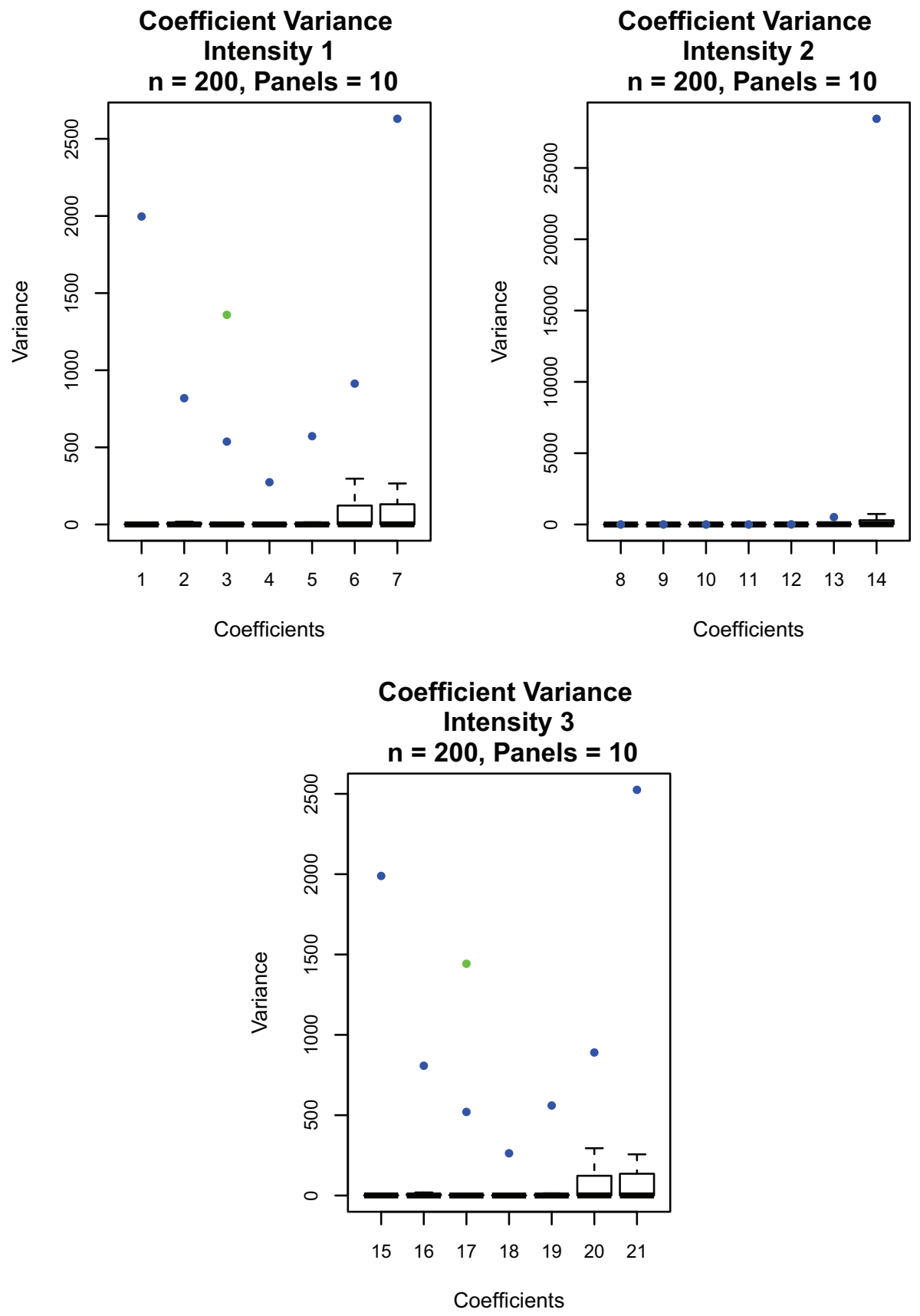

Figure 10: Sample and model based estimates of the variance of the B-Spline coefficients. The model based estimates of the variance are presented as boxplots, and their means are given by the green dots. The sample based estimates are shown as blue dots. 

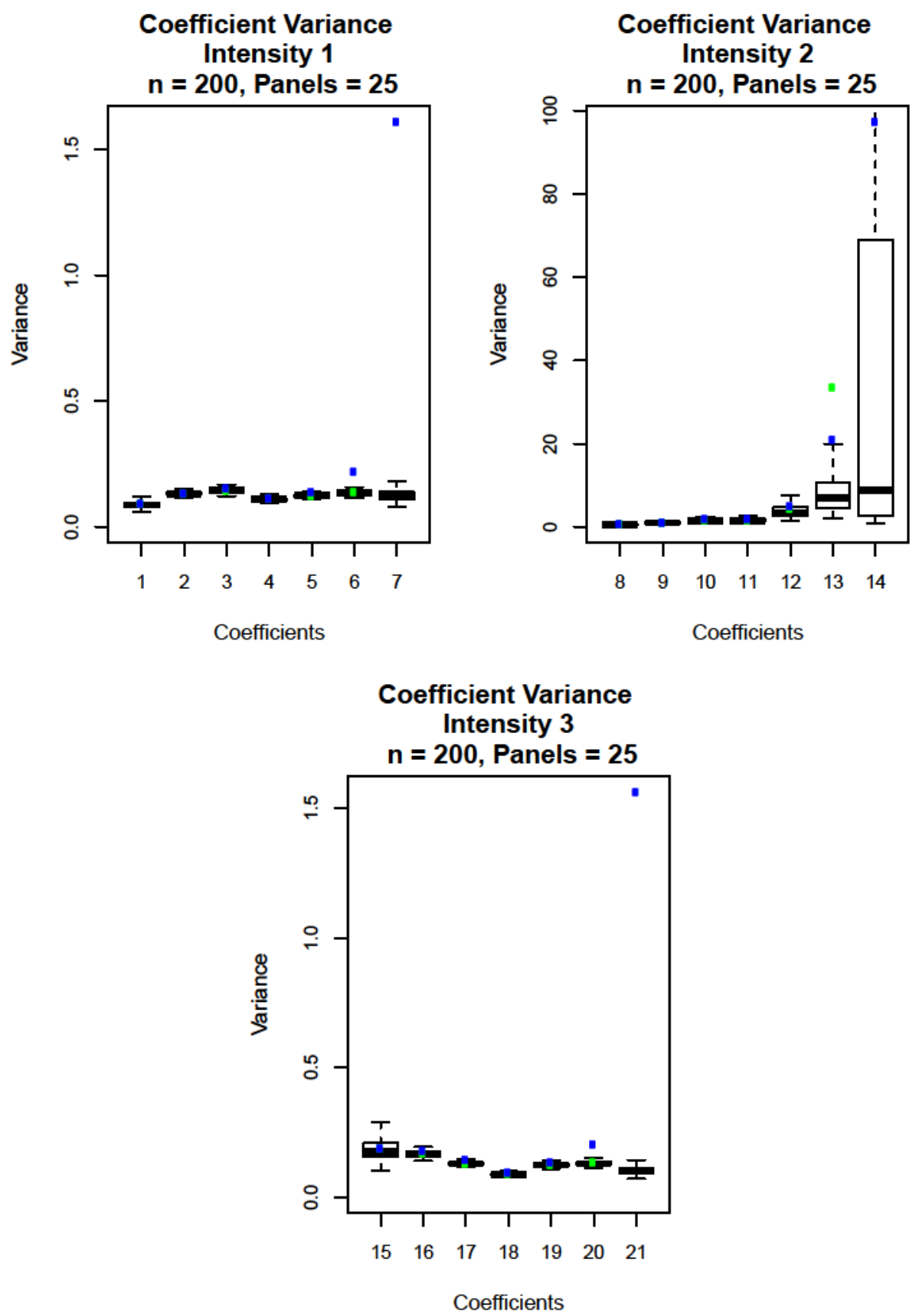

Figure 11: Sample and model based estimates of the variance of the B-Spline coefficients. The model based estimates of the variance are presented as boxplots, and their means are given by the green dots. The sample based estimates are shown as blue dots. 

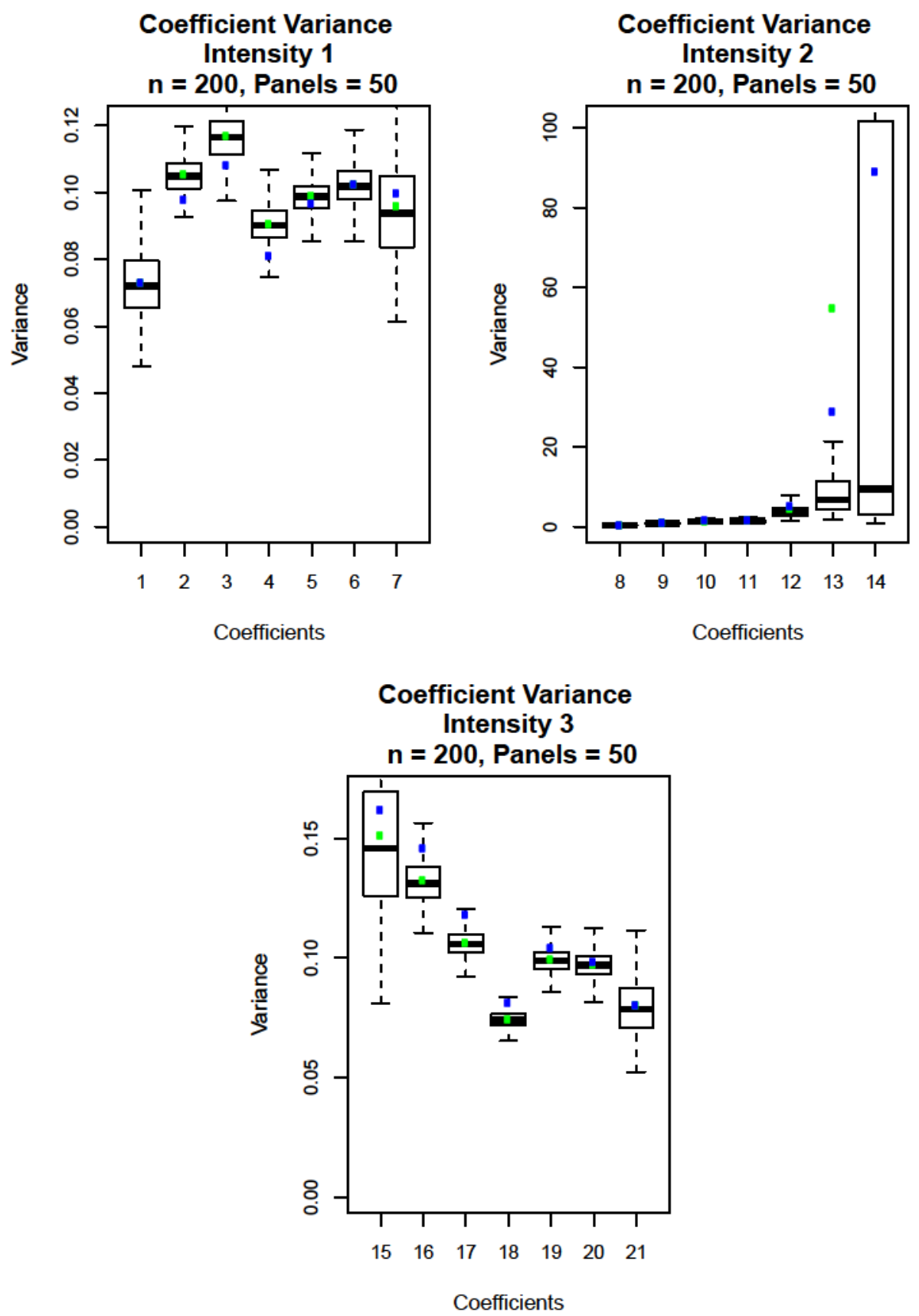

Figure 12: Sample and model based estimates of the variance of the B-Spline coefficients. The model based estimates of the variance are presented as boxplots, and their means are given by the green dots. The sample based estimates are shown as blue dots. 

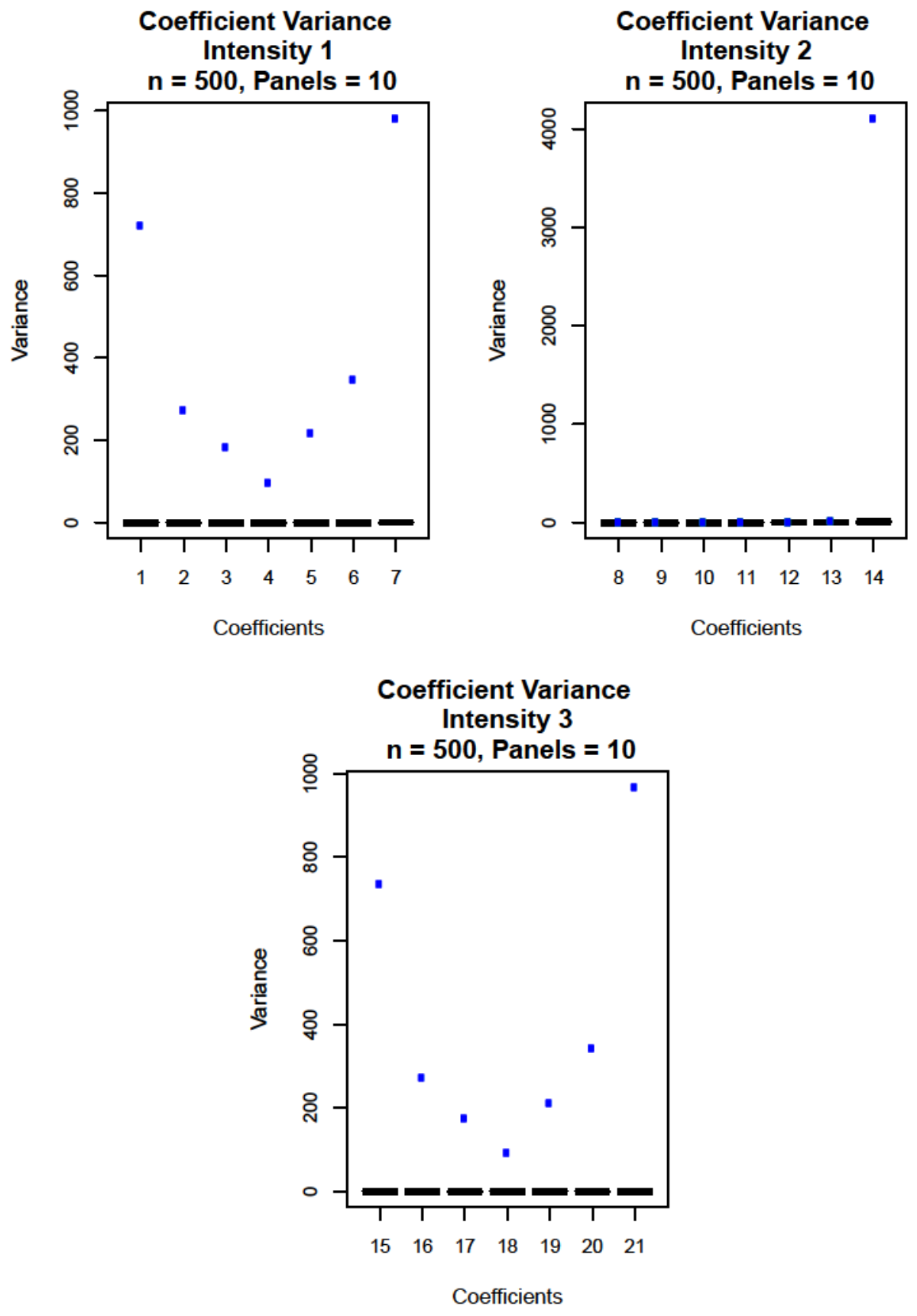

Figure 13: Sample and model based estimates of the variance of the B-Spline coefficients. The model based estimates of the variance are presented as boxplots, and their means are given by the green dots. The sample based estimates are shown as blue dots. 

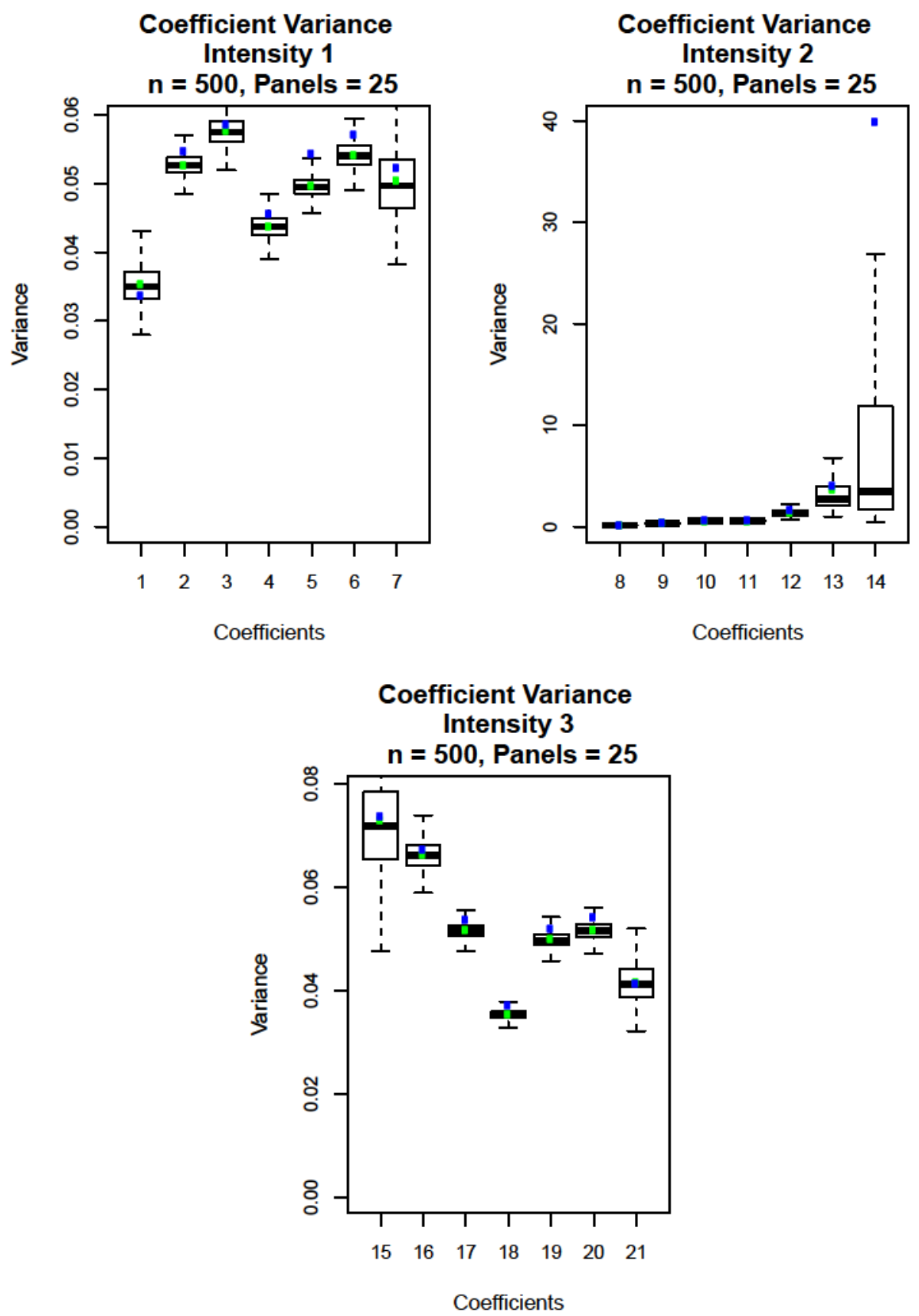

Figure 14: Sample and model based estimates of the variance of the B-Spline coefficients. The model based estimates of the variance are presented as boxplots, and their means are given by the green dots. The sample based estimates are shown as blue dots. 

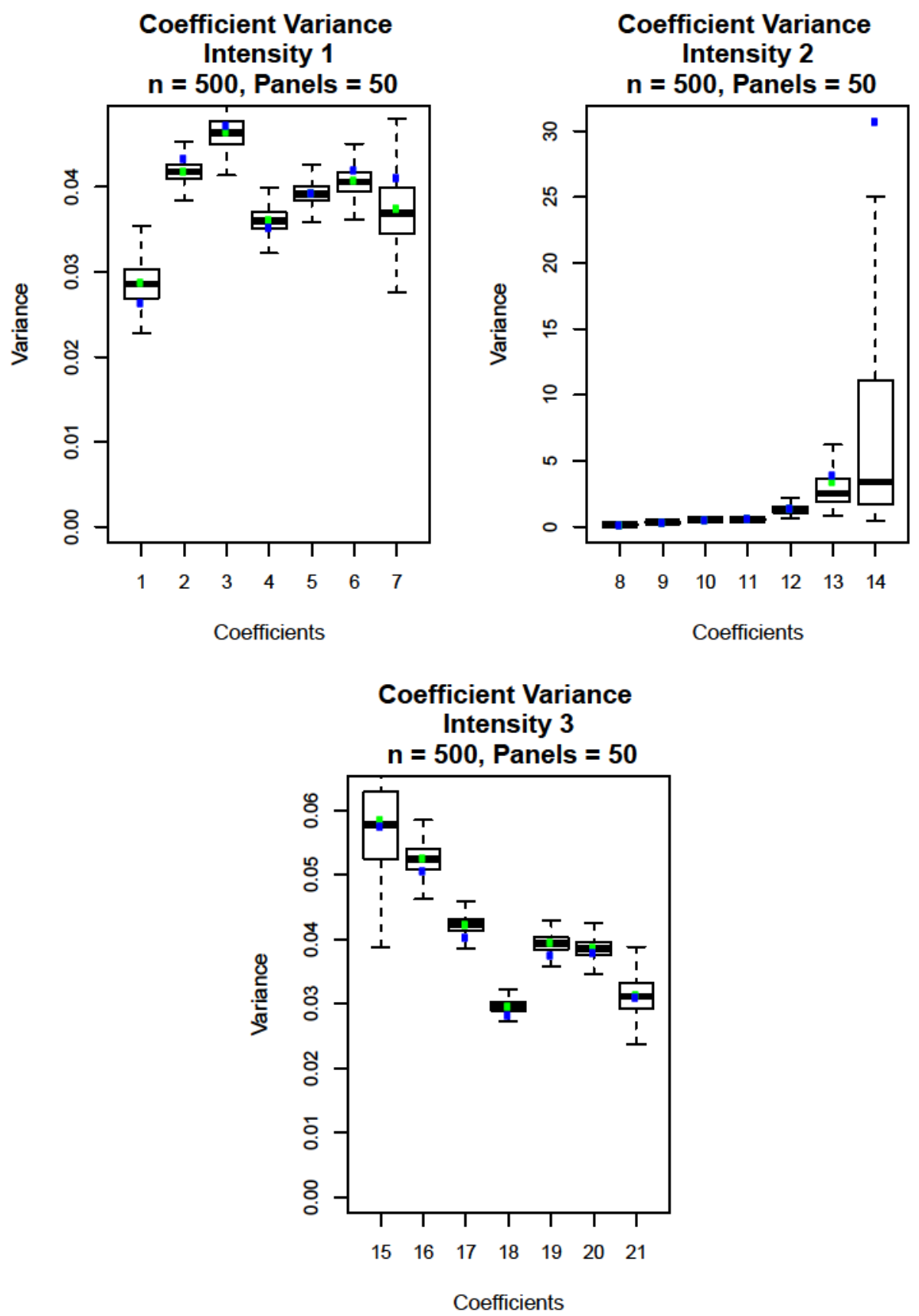

Figure 15: Sample and model based estimates of the variance of the B-Spline coefficients. The model based estimates of the variance are presented as boxplots, and their means are given by the green dots. The sample based estimates are shown as blue dots. 
panels or individuals. For the 10 panel cases, presented in Figures 4 and 7, the effect of this bias is particularly visible for intensity 3 , where the mean estimate seems to follow that of intensity 1 , yet the median estimate is close to the true function.

Despite these differences, the model based estimates of the variance resemble their sample based counterparts ${ }^{3}$, as shown in Figures 10-15. Note that the 10 panel cases, shown in Figures 4 and 7 are particularly troublesome. Due to extreme values, the mean variance estimates are quite large and are often beyond the upper bound of the graph. However, for the 25 and 50 panel cases, they are very close to the sample based estimates and are relatively well behaved. Overall, the performance of the variance estimators indicate that the model based estimates are good when the data provides enough information to capture the features of the underlying function. Further improvements to the estimation procedure, may contribute to eliminating bias, particularly with regards to multiple transitions within panels.

\subsubsection{P-Splines}

The simulation data described in Section 4.1 may be fit using cubic P-Spline based models. When fitting P-Splines, the number of knots generally exceeds the number of data points and the penalty term imposes the appropriate fit. For practical purposes, specifically estimation time, the number of knots has been arbitrarily limited to 40 . This produces three scenarios, where the number of knots is greater than, lower than, or comparable to the number of panels.

Unlike in the B-Spline case, optimizing P-Spline based models focuses less on the number and location of knots, than on the smoothing parameter associated with the range, such as in the case of natural splines, edge effects will usually introduce some amount of bias.

${ }^{3}$ The sample based variance estimators are obtained by computing the variance of the coefficient estimates over all simulated data sets. 
penalty term. Selection of the smoothing paramater, $\vec{\lambda}=\left\{\lambda_{1}, \lambda_{2}, \lambda_{3}\right\}$, is based on the Bayesian Information Criterion (BIC), as detailed in Sections 2.6 and 3.6. Given that the BIC is a usually well behaved non-linear function, our optimization scheme uses a brute force algorithm to obtain approprite values.

Unfortunately, the brute force method is a rather lengthy process and time constraints have impeeded us from optimizing the smoothing parameter for every simulated data set. Since our primary interest lies in the estimation of P-Spline coefficients and not in the smoothing parameter itself (considered a nuisance parameter), estimates of the $\lambda_{i}$ 's have been produced using a much smaller sample for each scenario described in Section 4.1. While the parameter estimates produced using the fixed $\lambda_{i}$ 's are not necessairly optimal, they are comparable, and should approximate the true functions reasonably well.

Based on the BIC's of the test sample, the $\lambda_{1}$ 's were generally around 300 , while the $\lambda_{2}$ 's and $\lambda_{3}$ 's were fixed at respectively $10^{4}$ and $10^{6}$. For scenarios with less panels and observations, $\lambda_{1}$ is somewhat larger, achieving even 5000 in the most extreme case. These values produced the results presented in Figures 16-27.

As shown in Figures 16-21, the P-Spline model approximates the underlying intensity functions reasonably well, however the accuracy of the estimation depends greatly on the number of panels and the smoothing parameter. For instance, the 10 panel cases used smoothing parameters that were larger than in other scenarios because their signal-to-noise ratio is much lower. For these cases, optimizing the BIC oversmoothed the data, producing a model that failed to properly capture the features of the intensities, and in particular intensity 1. 

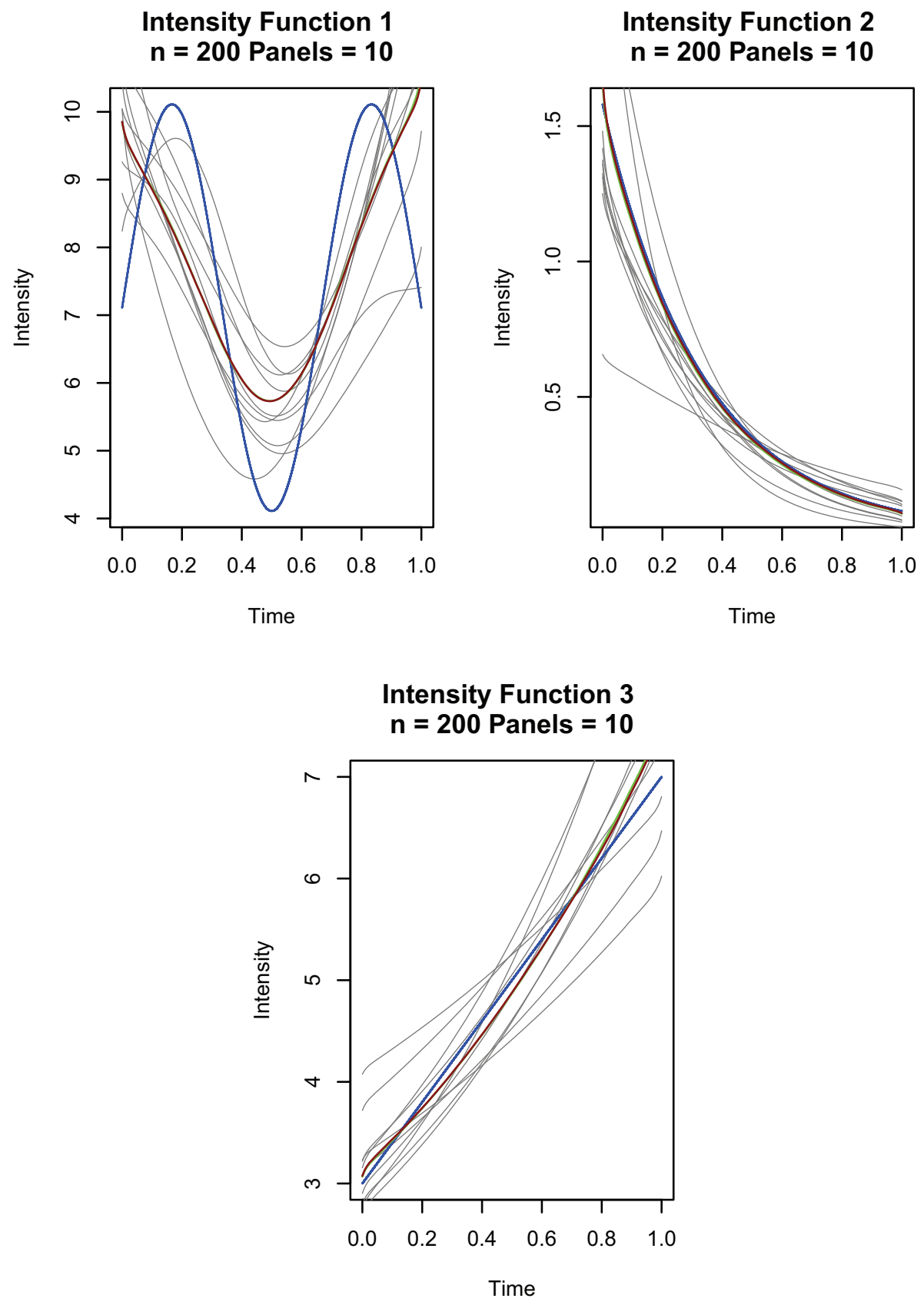

Figure 16: P-Spline based estimates of simulated data described in Section 4.1 with a cubic basis and 40 equidistant knots. The true, mean and median values are respectively represented by the blue, green and red lines. A sample of fits are also show in grey. 

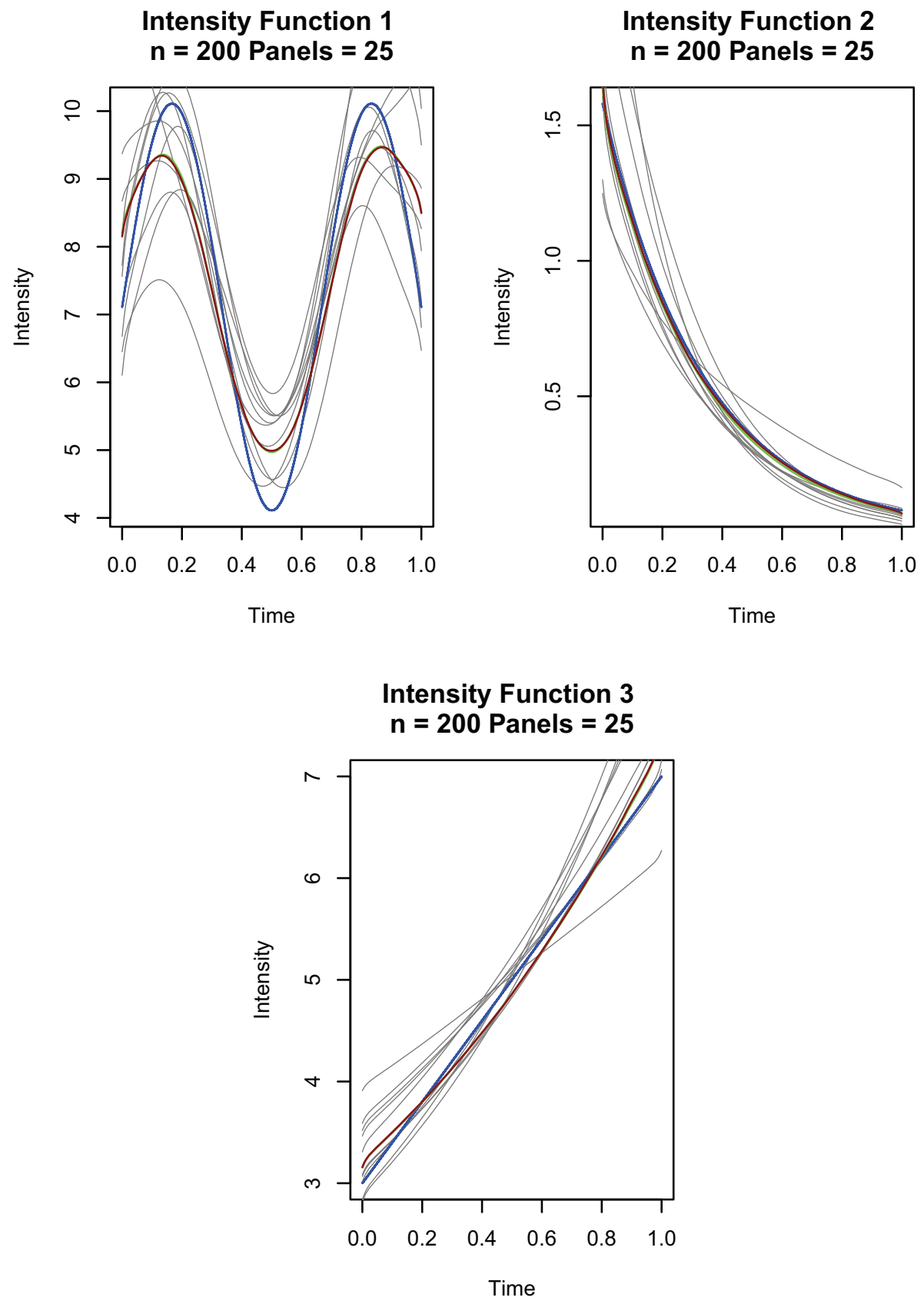

Figure 17: P-Spline based estimates of simulated data described in Section 4.1 with a cubic basis and 40 equidistant knots. The true, mean and median values are respectively represented by the blue, green and red lines. A sample of fits are also show in grey. 

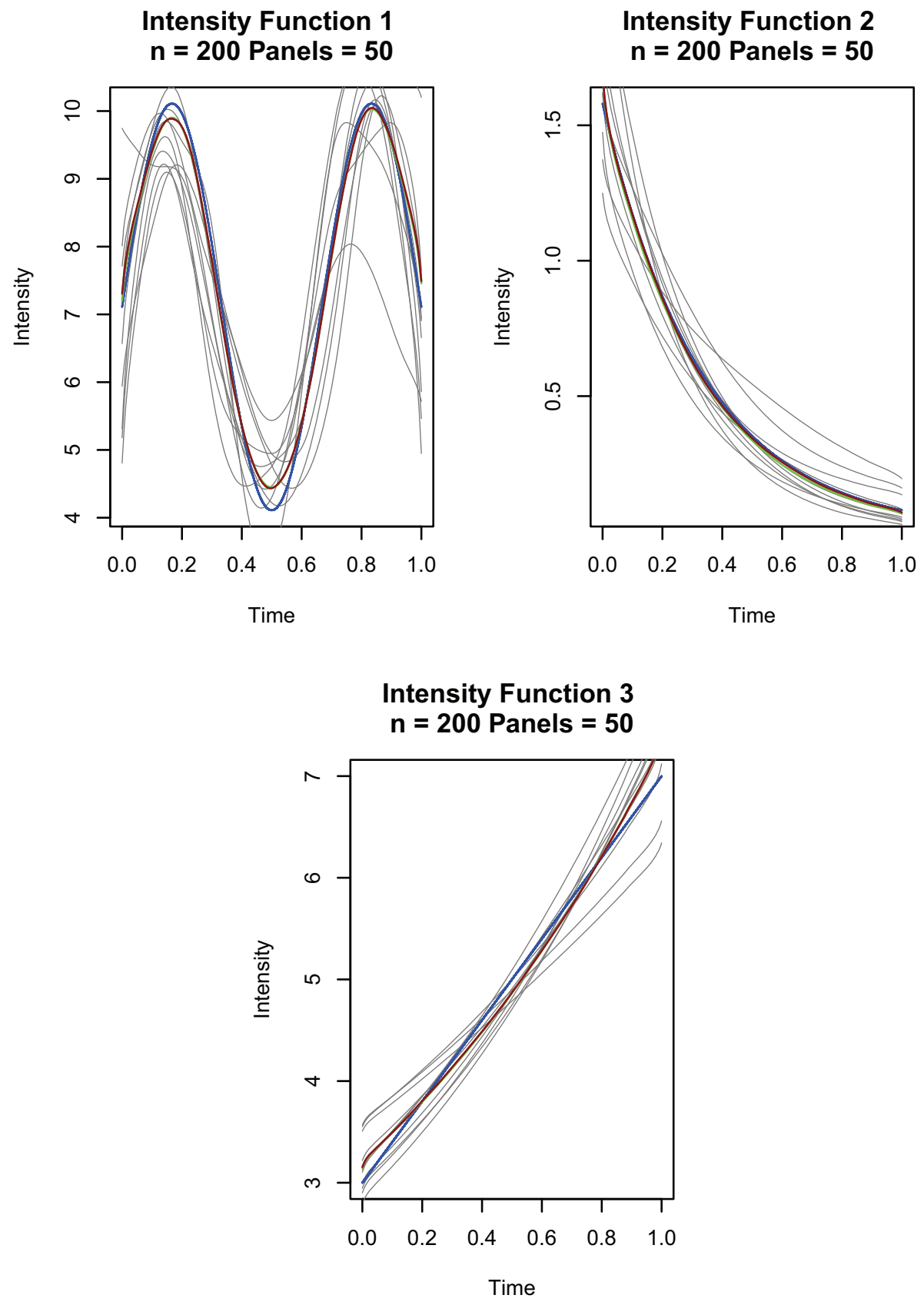

Figure 18: P-Spline based estimates of simulated data described in Section 4.1 with a cubic basis and 40 equidistant knots. The true, mean and median values are respectively represented by the blue, green and red lines. A sample of fits are also show in grey. 

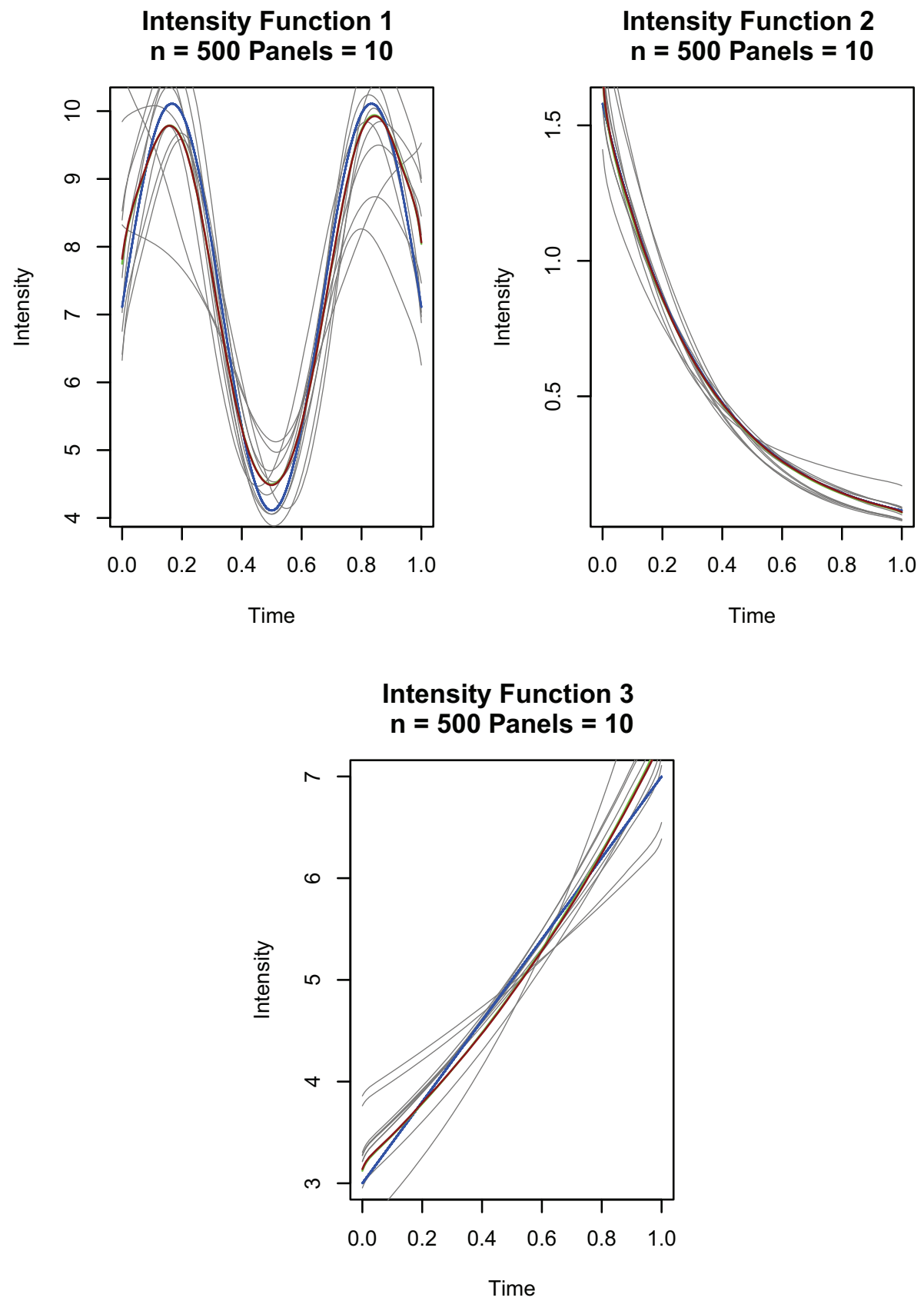

Figure 19: P-Spline based estimates of simulated data described in Section 4.1 with a cubic basis and 40 equidistant knots. The true, mean and median values are respectively represented by the blue, green and red lines. A sample of fits are also show in grey. 

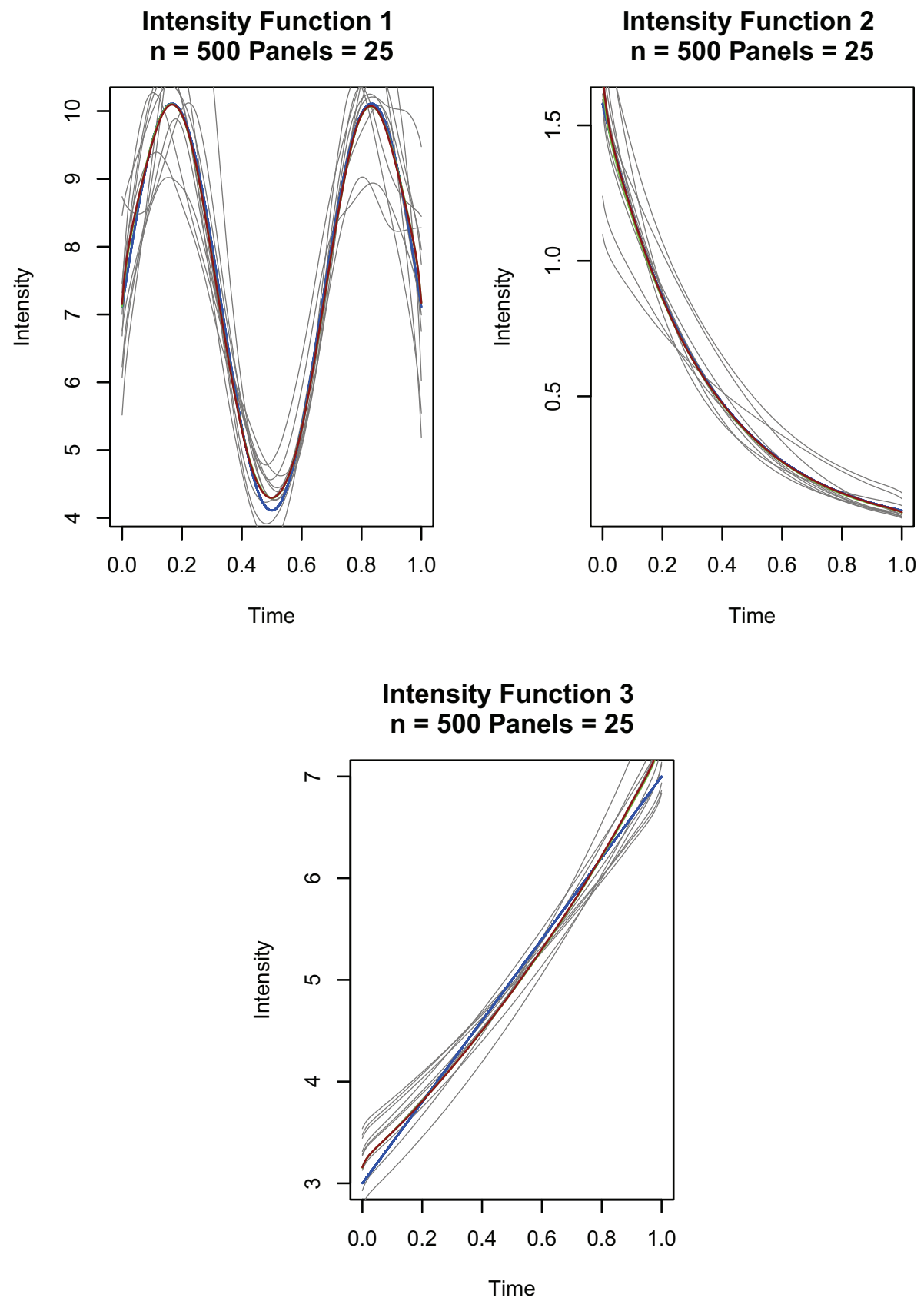

Figure 20: P-Spline based estimates of simulated data described in Section 4.1 with a cubic basis and 40 equidistant knots. The true, mean and median values are respectively represented by the blue, green and red lines. A sample of fits are also show in grey. 

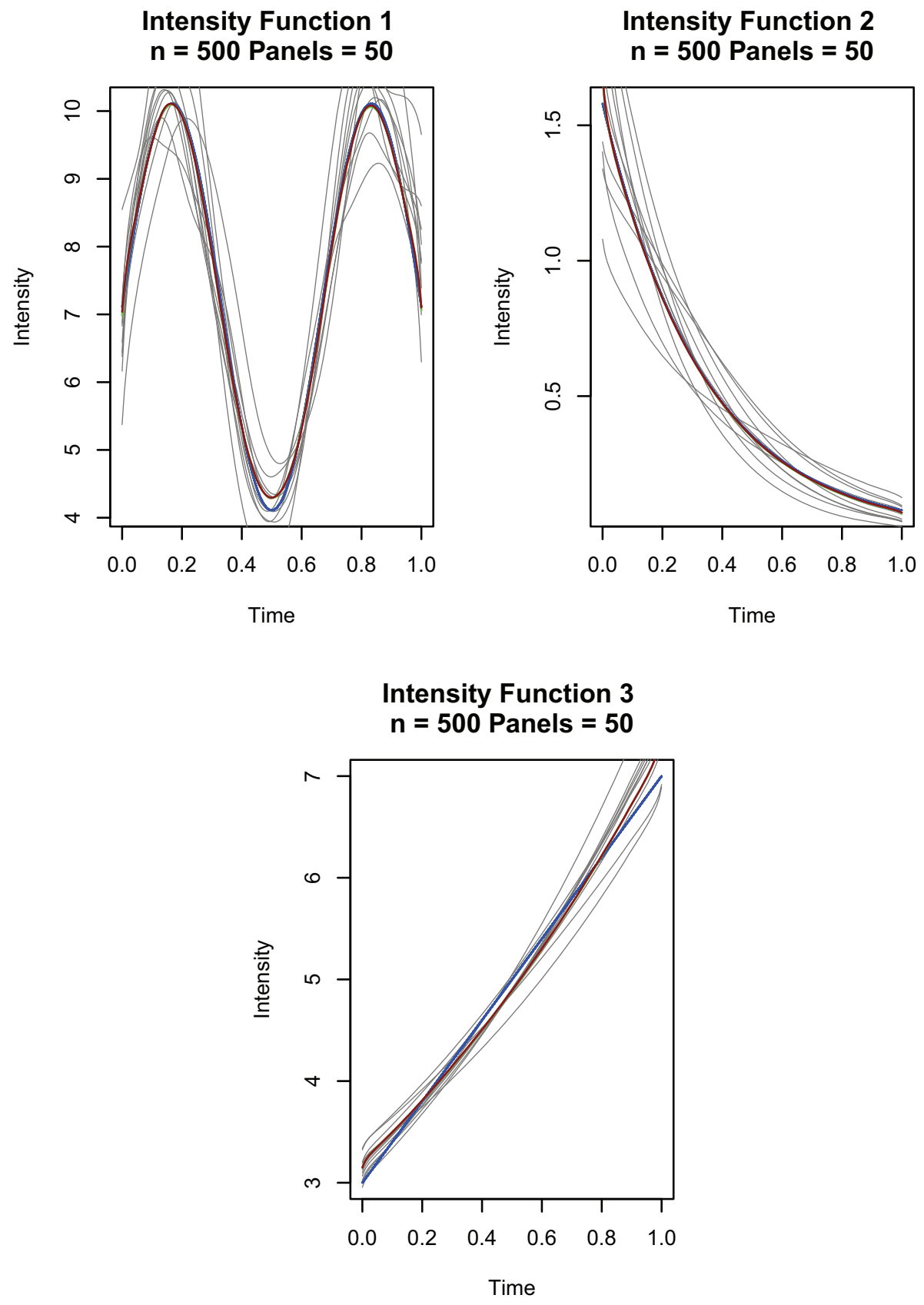

Figure 21: P-Spline based estimates of simulated data described in Section 4.1 with a cubic basis and 40 equidistant knots. The true, mean and median values are respectively represented by the blue, green and red lines. A sample of fits are also show in grey. 

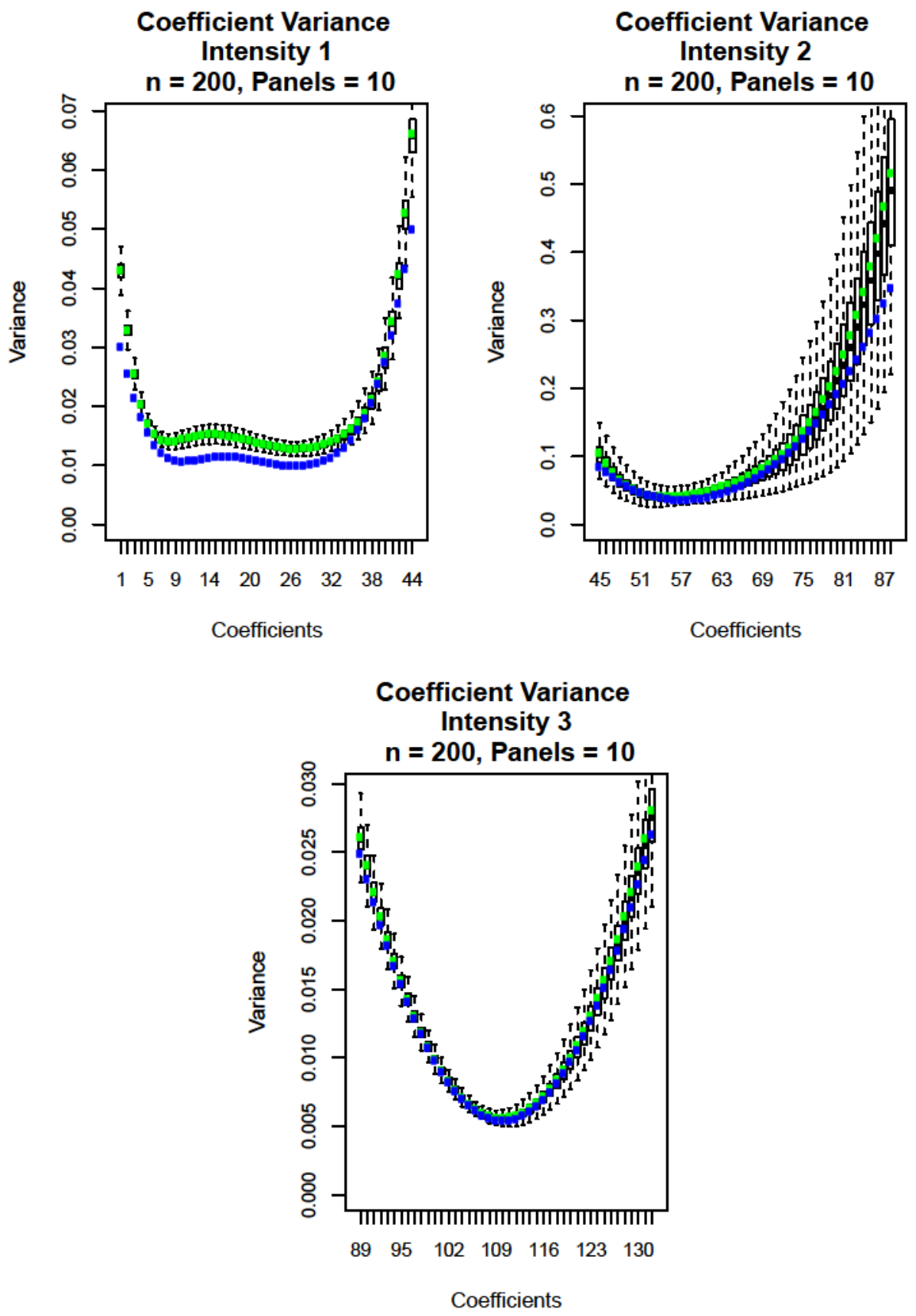

Figure 22: Sample and model based estimates of the variance of the P-Spline coefficients. The model based estimates of the variance are presented as boxplots, and their means are given by the green dots. The sample based estimates are shown as blue dots. Coefficients 1-44 are associated with intensity 1, 45-88 with intensity 2 , and 89-132 with intensity 3 . 

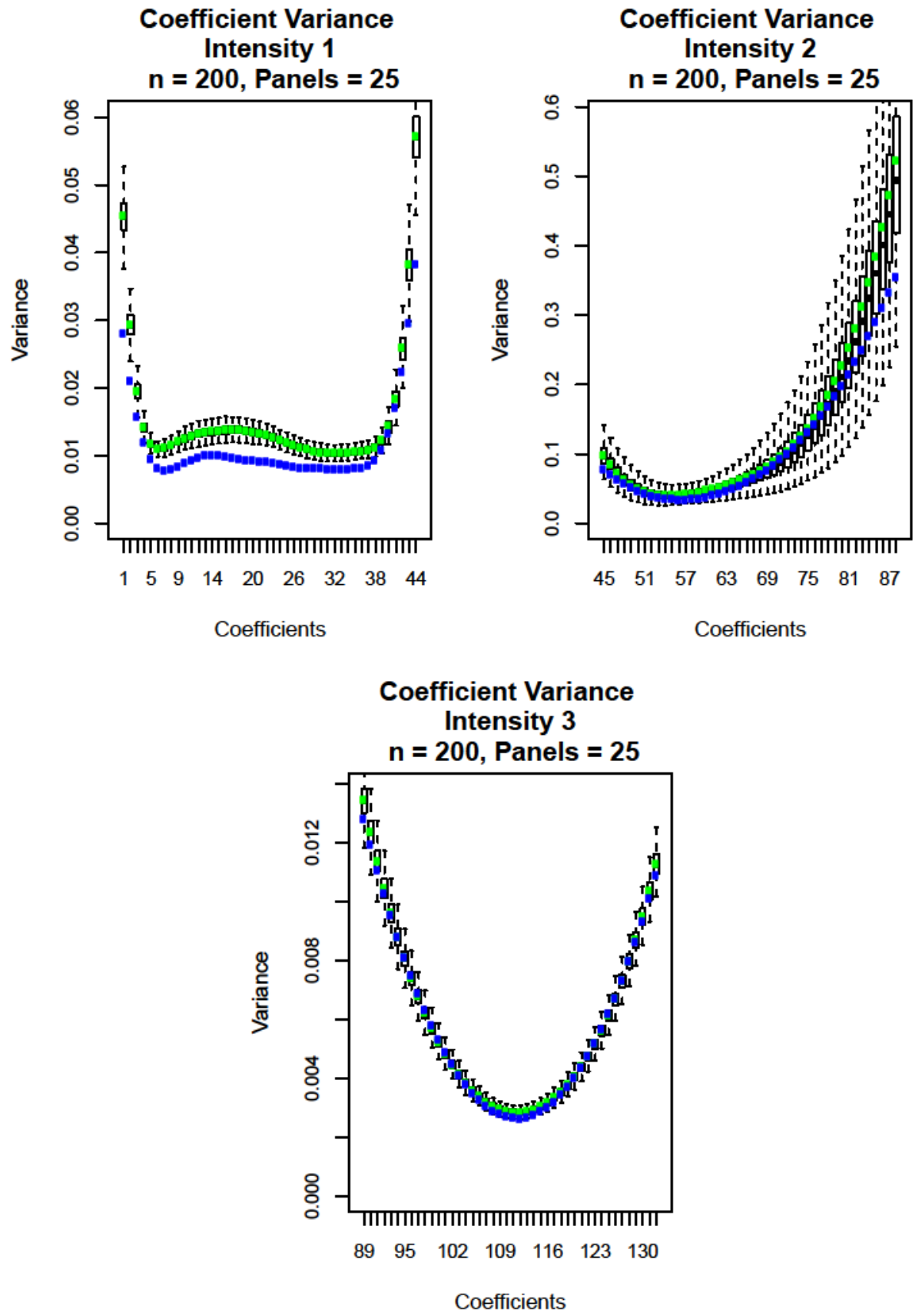

Figure 23: Sample and model based estimates of the variance of the P-Spline coefficients. The model based estimates of the variance are presented as boxplots, and their means are given by the green dots. The sample based estimates are shown as blue dots. Coefficients 1-44 are associated with intensity 1, 45-88 with intensity 2 , and 89-132 with intensity 3 . 

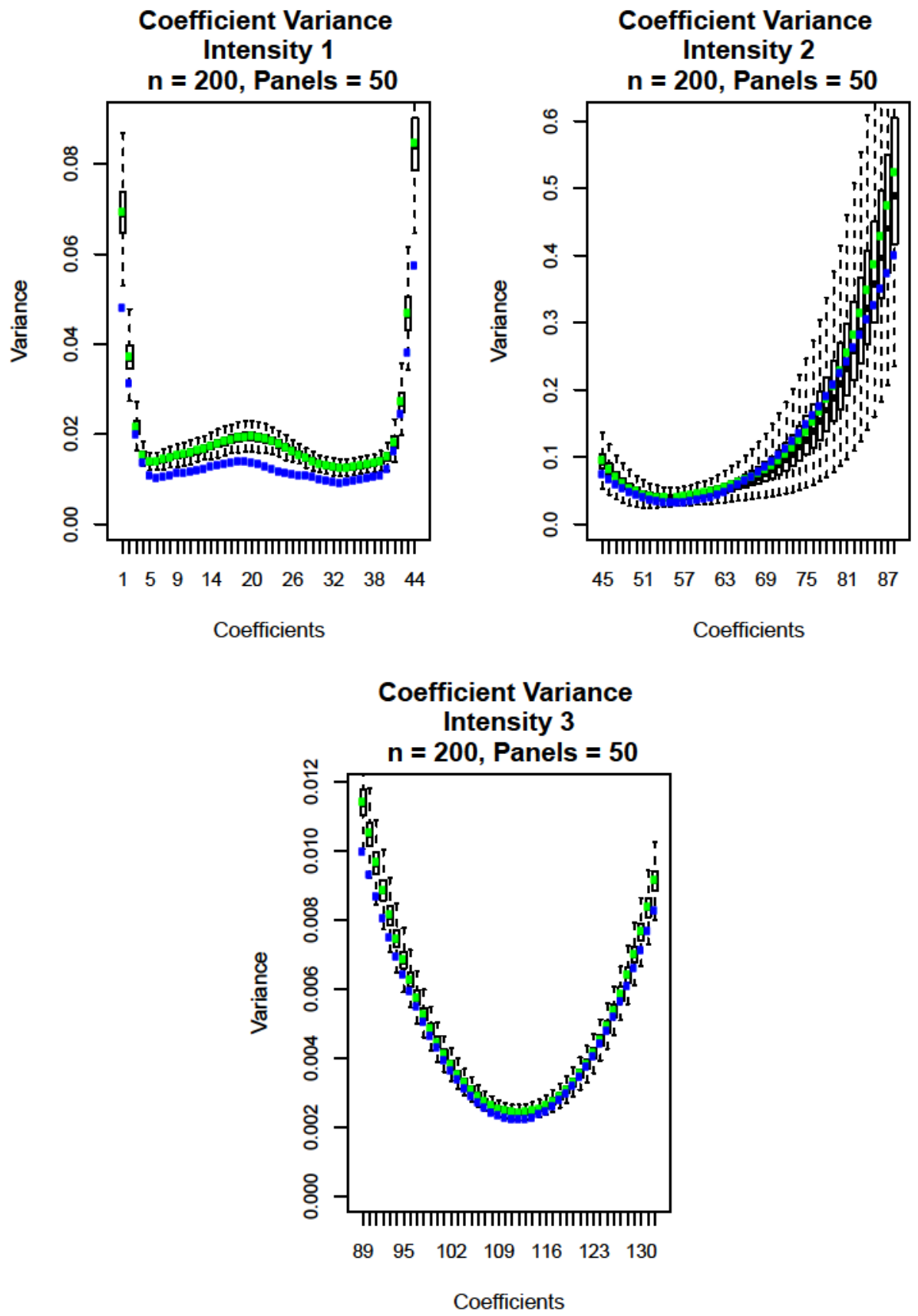

Figure 24: Sample and model based estimates of the variance of the P-Spline coefficients. The model based estimates of the variance are presented as boxplots, and their means are given by the green dots. The sample based estimates are shown as blue dots. Coefficients 1-44 are associated with intensity 1, 45-88 with intensity 2 , and 89-132 with intensity 3 . 

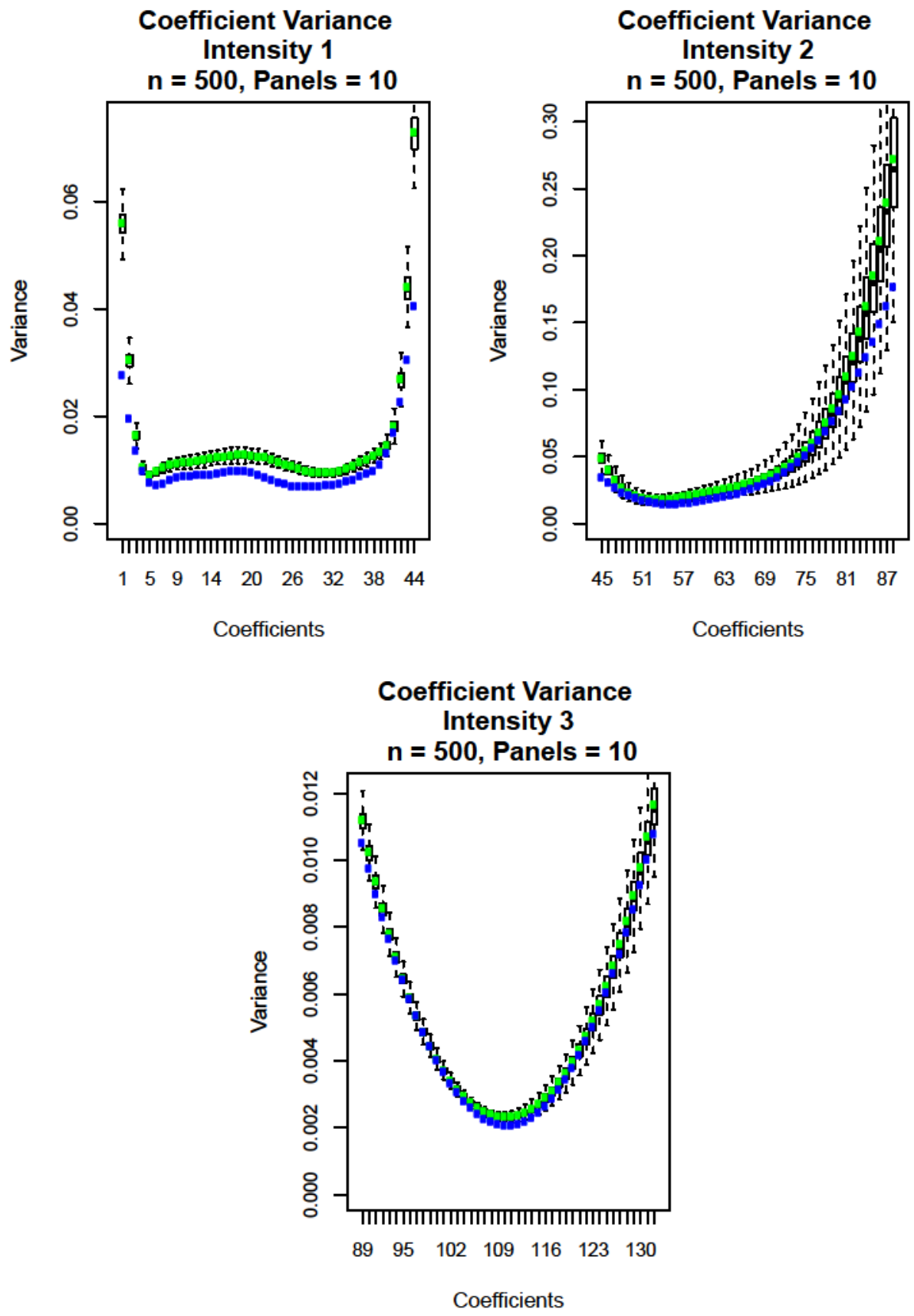

Figure 25: Sample and model based estimates of the variance of the P-Spline coefficients. The model based estimates of the variance are presented as boxplots, and their means are given by the green dots. The sample based estimates are shown as blue dots. Coefficients 1-44 are associated with intensity 1, 45-88 with intensity 2 , and 89-132 with intensity 3 . 

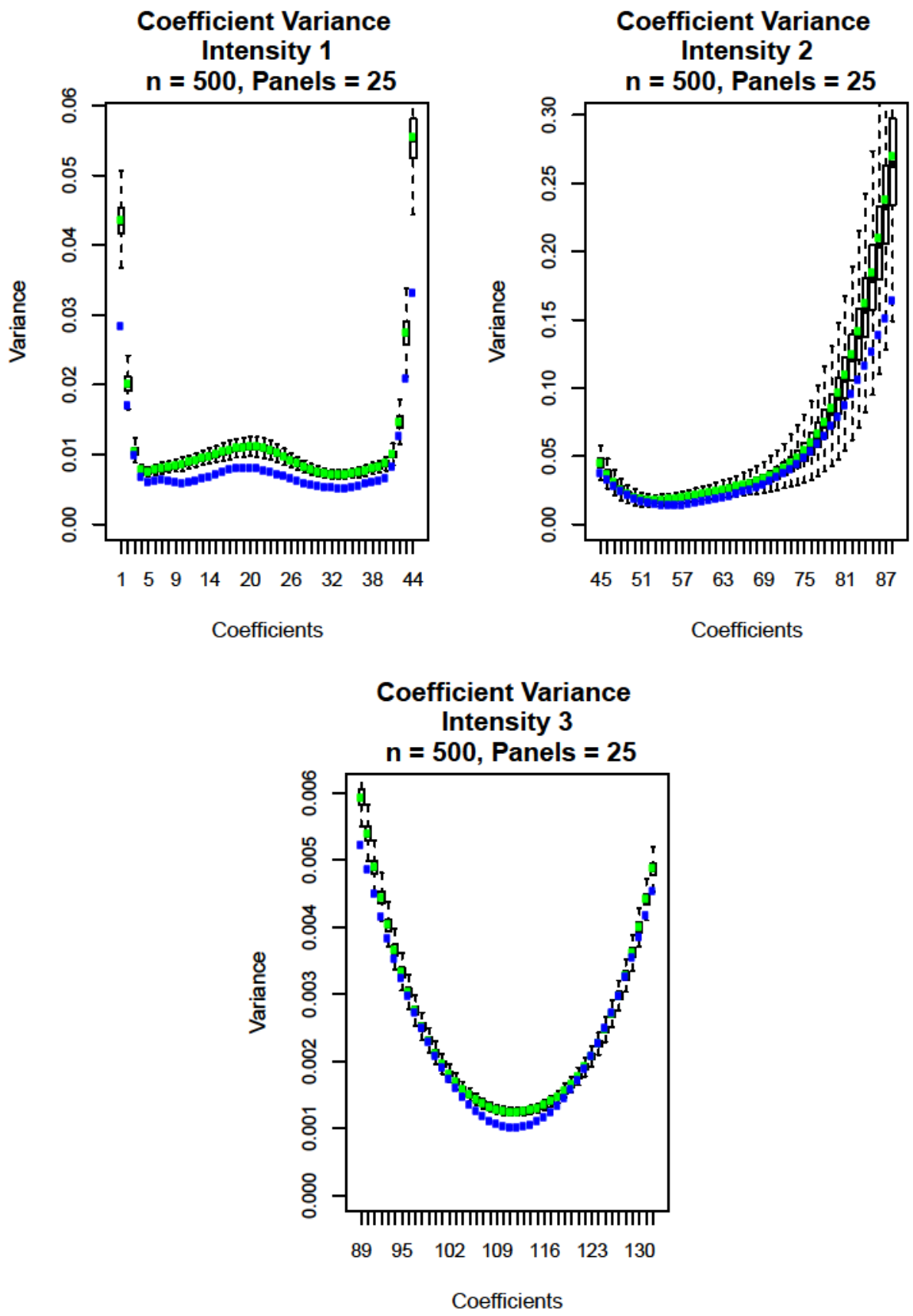

Figure 26: Sample and model based estimates of the variance of the P-Spline coefficients. The model based estimates of the variance are presented as boxplots, and their means are given by the green dots. The sample based estimates are shown as blue dots. Coefficients 1-44 are associated with intensity 1, 45-88 with intensity 2 , and 89-132 with intensity 3 . 

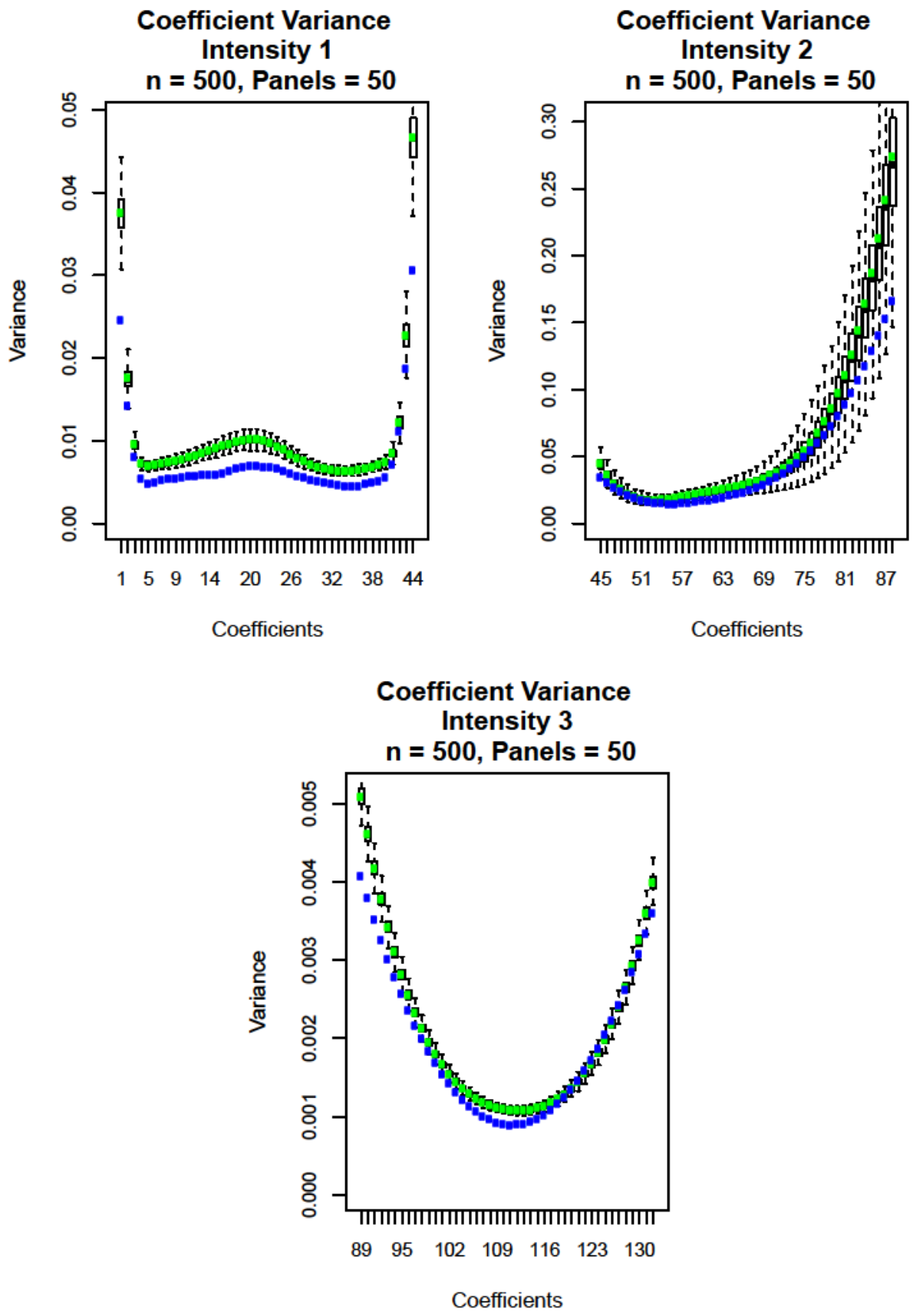

Figure 27: Sample and model based estimates of the variance of the P-Spline coefficients. The model based estimates of the variance are presented as boxplots, and their means are given by the green dots. The sample based estimates are shown as blue dots. Coefficients 1-44 are associated with intensity 1, 45-88 with intensity 2 , and 89-132 with intensity 3 . 
While the major differences between Figures 16-21 may be attributed to the smoothing parameter, the selection of this parameter is affected by the sample size. If the optimization procedure had been applied to every simulated data set, or if the training data had been larger, it is likely that the 10 panel cases would have produced better results. However, it is probable that the samples with 500 individuals would still outperform the samples with 200 individuals, due to the larger empirical evidence used during the parameter selection process. In other words, larger sample sizes produce more appropriate $\lambda$ 's, which lead to models that more accurately reflect the true transition intensities.

Figures 22-27 show the model and sample based variance estimates of the spline coefficients, however none of these reflect the variability inherent with the selection of the smoothing parameter. This bias aside, the variance estimates are relatively close, indicating that the model based variance estimates are working well under the scenarios considered.

\subsection{Examples}

Applying the techniques proposed in Section 3 to previously modeled data may further illustrate their use. For example, consider the dataset with covariates proposed by Kalbfleisch and Lawless (1985), and the illness-death simulation examined by Titman (2011).

\subsubsection{Kabfleisch \& Lawless}

The Kalbfleisch and Lawless data is a three state model characterized by the constant transition intensity matrix $\boldsymbol{Q}_{0}$, shown below ${ }^{4}$. Note that subjects can move

\footnotetext{
${ }^{4}$ In Kalbfleisch and Lawless's publication, $\beta_{1}$ is witten as $\beta_{3}$, however this is an obvious typographical error since it is referred to as $\beta_{1}$ throughout the remainder of their article.
} 
Table 1: Parameter Estimates and True Values

\begin{tabular}{cccc} 
Parameter & True Value & MLE & Estimated SE \\
\hline$\alpha_{12}$ & -2.30 & -2.177 & 0.356 \\
$\beta_{1}$ & 0.50 & 0.700 & 0.406 \\
$\beta_{2}$ & -0.50 & -0.772 & 0.406 \\
$\alpha_{13}$ & -2.30 & -2.659 & 0.235 \\
$\alpha_{21}$ & -1.90 & -1.389 & 0.278 \\
$\beta_{3}$ & 0.50 & 0.284 & 0.307 \\
$\beta_{4}$ & 0.50 & 0.111 & 0.304 \\
$\alpha_{23}$ & -2.30 & -2.246 & 0.245 \\
\hline
\end{tabular}

Source: Kalbfleisch and Lawless, 1985, Table 2, pg. 869

freely between states 1 and 2, whereas state 3 is absorbing. The authors propose a time homogeneous model using constant intensity functions with four covariate levels, parameterized by $\vec{z}=\left(z_{1}, z_{2}\right)$, for $z_{1} \in(0,1)$ and $z_{2} \in(0,1)$. The true $\mathrm{e}^{5}$ and estimated parameters for the time homogeneous model are presented in Table 1.

$$
\boldsymbol{Q}_{0}=\left(\begin{array}{ccc}
q_{11}(\vec{z}) & e^{\alpha_{12}+z_{1} \beta_{1}+z_{2} \beta_{2}} & e^{\alpha_{31}} \\
e^{\alpha_{21}+z_{1} \beta_{3}+z_{2} \beta_{4}} & q_{22}(\vec{z}) & e^{\alpha_{23}} \\
0 & 0 & 0
\end{array}\right)
$$

Applying the methods from Section 3 to the Kalbfleisch and Lawless data yields the results presented in Figure 28. The solid lines represent the fit using the P-Spline based method, the dashed lines are the true values, and the dotted lines are the maximum likelihood estimates for the homogeneous case, as presented in Table 1.

\footnotetext{
${ }^{5}$ The Kalbfleisch and Lawless data is a simulated data set based on the matrix $\boldsymbol{Q}_{0}$. The values used to generate the data are assumed to be true.
} 

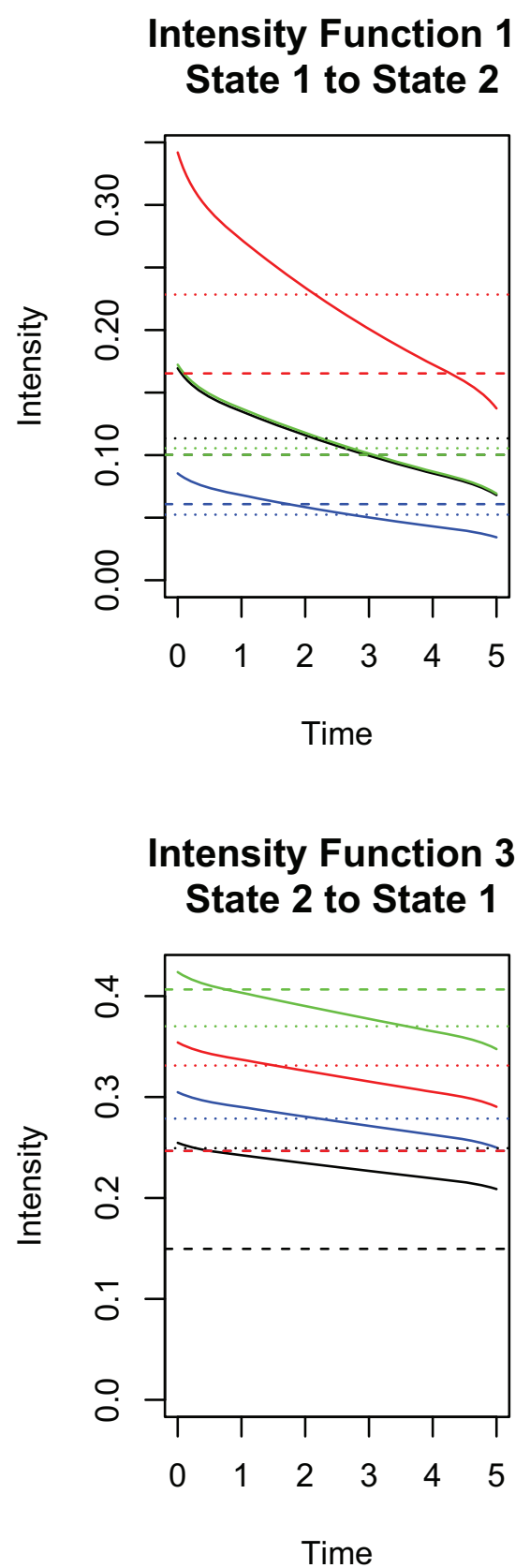

\section{Intensity Function 2} State 1 to State 3

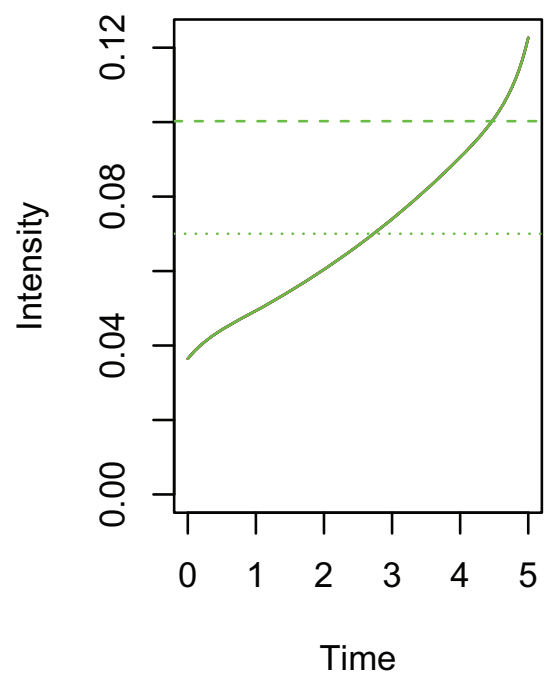

Intensity Function 4 State 2 to State 3

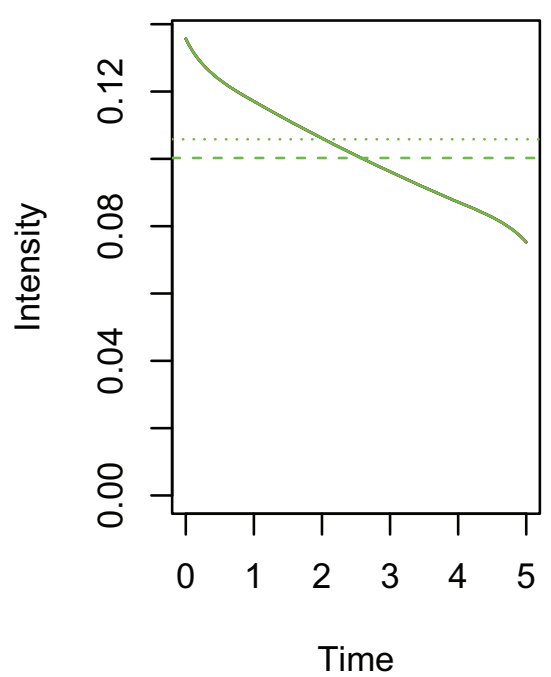

Figure 28: True and estimated transition intensities for the Kalbfleisch and Lawless data. The solid line is the fit for the P-Spline based model, and the dashed and dotted lines are repsectively the true and estimated values for the constant intensity model. Each covariate level is represented by a color. 
Each of the covariate levels, $\vec{z}=(0,0), \vec{z}=(0,1), \vec{z}=(1,0)$, and $\vec{z}=(1,1)$, is represented by a different color line, repsectfully: black, blue, red and green.

Since the data is based on constant intensities, the optimal value for the smoothing parameter, $\lambda$, is near infinity. The resulting model attains the minimum of 12 effective degrees of freedom under the P-Spline penalty (2 per spline and 1 per covariate). Consequently, the model is equivilant to a linear regression with covariates, in the exponential scale.

As shown in Figure 28, the P-Spline intensities are on par with the with the maximum likelihood estimates for the homogeneous case. In effect, the P-Spline mean estimates over $t$ are slightly closer to the true values than the Kalbfleisch and Lawless MLE's, however this minute difference comes at the expense of an extra degree of freedom for each intensity.

\subsubsection{Titman}

The simulated dataset examined by Titman (2011) uses a three state health-illness model, which considers individuals as healthy (state 1), ill (state 2), or dead (state 3). Like in previously examined models, state 3 is absorbing, whereas state 1 and 2 are not. All individuals are considered healthy at the initial starting time, $t=0$.

As mentioned briefly in Section 3.8, there are several ways to model nonhomogeneous Markov processes. Titman proposes a method based on solving the Kolmogorov Forward Equations (KFE), presented in (2.4), using a system of first order differential equations (ODE).

Unlike previously modeled datasets, the Titman data is collected at random intervals for each individual. The data has been adapted through binning, coarsening 
observations into discrete time points. We have arbitrairly chosen 16 equidistant sample times over $t=[0,13]$, such that there is at least one transition in each panel.

The data set also contains two covariates. One is dichotomous and the other is continuous. For simplicity, only the discrete covariate has been included in the model. Readers interested in the use continuous type covariates in non-homogeneous Markov models should consult Titman (2011), and in particular the web appendix which provides more detailed examples of the use of both discrete and continuous type covariates.

Applying the techniques outlined in Section 3 to the Titman data produces the results shown in Figure 29. The P-Spline approximations are presented as solid lines, and the equivilant estimates using Titman's model are displayed as dashed lines.

Figure 29 also gives the impression that the Titman model is more stable than it's P-Spline counterpart. This effect is mainly due to the Titman model having only 11 degrees of freedom and much stronger assumptions ${ }^{6}$, while the P-Spline model has approximately 34 effective degrees of freedom and is non-parametric. Here, we suspect that our approach to selecting $\lambda$ requires further investigation.

Overall, the P-Spline based model estimates the shape of the Titman transition intensities reasonably well, however their amplitude is often underestimated. This could very well be a direct result of the binning process, which reduces the amount of available information, as well as the low number of observable transitions near the extremities of the data.

\footnotetext{
${ }^{6}$ The Titman model attributes four terms to covariates, four to constant intensities, and three to the B-Spline that modulates the non-homogeneous effect for the state 1 to state 2 transition.
} 

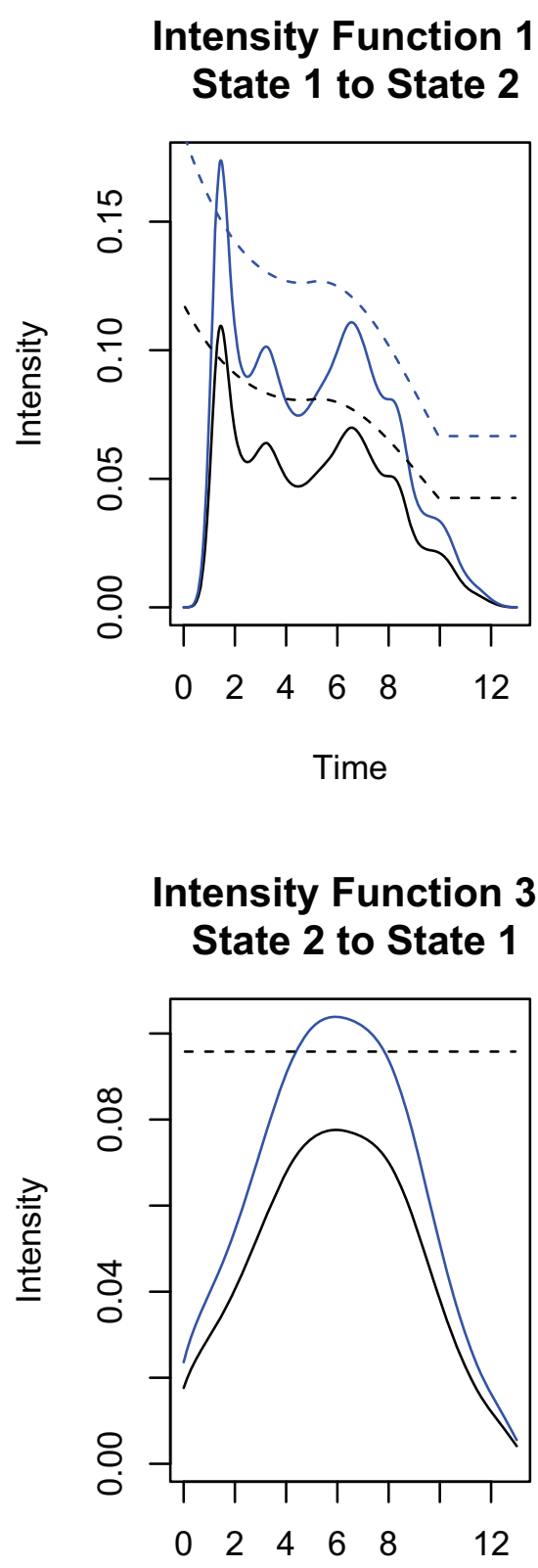

Time
Intensity Function 2 State 1 to State 3

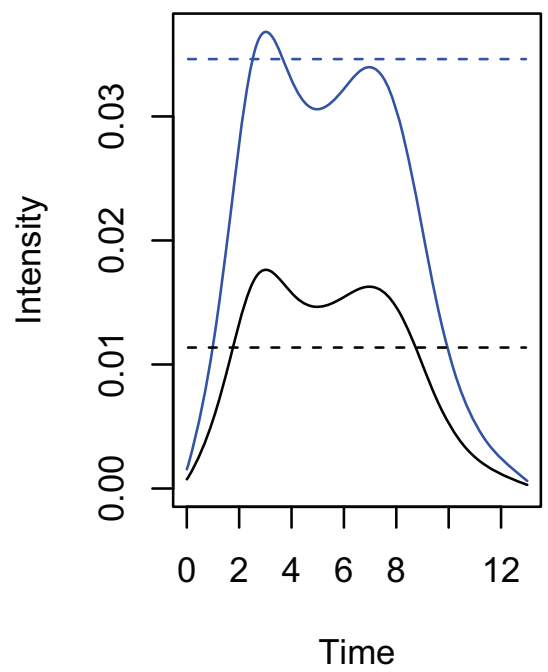

Intensity Function 4 State 2 to State 3

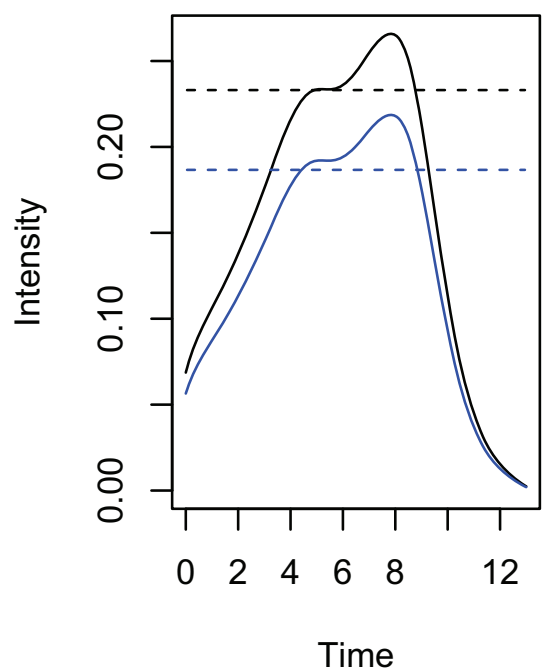

Figure 29: Estimated transition intensities for the Titman data. The solid lines are the P-Spline estimates with a different color for each covariate level, and the dotted lines are the corresponding values estimated using the Titman model. 


\section{Chapter 5}

\section{Conclusion}

Through the previous sections, we have outlined the basic theory for analyzing panel data under a Markov assumption with splines. This rather simple, yet flexible, structure may be easily modified or adapted for particular models or data. For the purpose of our discussion, we have limited ourselves to cubic splines and the P-Spline penalty proposed by Eilers and Marx (1996). However one need not be limited by these choices. Likewise, the Bayesian information criterion is only one out of many possible measures that may be used for indentifying optimal fits.

The combination of various penalties or basis, producing parametric, nonparametric, or even semi-parametric models, will eventually lead to better optimization strategies. The current implementation suffers from very long computation times due to the large number of numerical integrations and fittings required to produce optimal $\lambda$ 's. The use of more efficient numerical procedures would greatly reduce to the method's execution time.

Despite room for improvement, the proposed method has much to offer to researchers and practitioners. The ability to fit almost any panel data set with relatively few assumptions is quite attractive, particularly in regards to exploratory analysis. 


\section{Bibliography}

U. Naryan Bhat. Elements of Applied Stochastic Processes. Wiley-Interscience, 1984.

Carl de Boor. A Practical Guide to Splines. Springer-Verlag, 1978.

Paul H. C. Eilers and Brian D. Marx. Flexible smoothing with B-splines. Statistical Science, 1996.

Paul H. C. Eilers and Brian D. Marx. Splines, knots, and penalties. Journal, 2010.

Trevor Hastie, Robert Tibshirani, and Jerome Friedman. The Elements of Statistical Learning. Springer, 2009.

J. D. Kalbfleisch and J. F. Lawless. The analysis of panel data under a markov assumption. Journal of the American Statistical Association, 1985.

R Core Team. R: A Language and Environment for Statistical Computing. R Foundation for Statistical Computing, Vienna, Austria, 2013. URL http://www.Rproject.org/. ISBN 3-900051-07-0.

J. O. Ramsay and B. W. Silverman. Functional Data Analysis. Springer, 2005.

Jim Ramsay, Giles Hooker, and Spencer Graves. Functional Data Analysis with $R$ and MATLAB. Springer, 2009.

Andrew C. Titman. Flexible nonhomogeneous markov models for panel observed data. Biometrics, 2011. 